\title{
STABILITY OF CHARACTER SUMS FOR POSITIVE-DEPTH, SUPERCUSPIDAL REPRESENTATIONS
}

\author{
STEPHEN DEBACKER AND LOREN SPICE
}

\begin{abstract}
We re-write the character formulæ of [4] in a form amenable to explicit computations in $p$-adic harmonic analysis, and use them to prove the stability of character sums for a modification of Reeder's conjectural positive-depth, unramified, toral supercuspidal L-packets [21].
\end{abstract}

\section{INTRODUCTION}

In [21, §6.6, p. 18], Reeder generalises his joint work with the first-named author on the depth-0, supercuspidal local Langlands correspondence [10, Theorem 4.5.3] to construct some candidates for positive-depth, supercuspidal L-packets. These consist of what could be called the unramified, toral supercuspidal representations. (In the notation of Definitions 4.1.1 and 4.1.2, they are the representations $\mathrm{Y}_{G^{\prime}}^{G} \pi^{\prime}$ arising from cuspidal pairs $\left(\mathbf{G}^{\prime}, \pi^{\prime}\right)$ in which $\mathbf{G}^{\prime}$ is an unramified torus.) He proves that these sets satisfy many of the properties expected of L-packets, but does not show that appropriate combinations of the characters in an L-packet are stable. In this paper, we use the character formulæ of [4] to demonstrate the appropriate stability, after replacing the construction of Adler-Yu used in [21, §3] by an appropriate 'twisted' analogue (Definition 5.4). We show that there are cases when this twist is necessary (Example 5.5). It is possible, though we have not yet explored this, that our twist bears some relation to the notion of rectifier introduced by Bushnell and Henniart [9. Definition 1]. In any case, recent work of Yu [30] suggests that a different lift of the Weil representation in the construction of [29, Proposition 4.6 and Theorem 11.5] would obviate the need for this ad hoc twist.

The paper proceeds as follows. In $\$ 2$ we establish some basic notation regarding the groups and fields that we consider. Of particular importance in this section is the 'reduced discriminant' (Definition 2.2.8), which we use to normalise characters and orbital integrals in Definition 4.1.6. In $₫ 3$, we recall the definitions of the Moy-Prasad filtrations of a $p$-adic group and its Lie algebra, and introduce some refinements $(3.2)$.

The meat of the paper is $\$ 4$ in which we re-cast the character formula of [4] in a form that is more suitable for our use and, we believe, for later explicit calculations in harmonic analysis. In this section, we handle a wide range of characters, not just the toral ones considered by Reeder; but our work inherits from [4] a 'compactness' condition (see Definition 4.1.1). We believe that Theorem 4.6.2 provides an idea of the shape of a formula for the character of an arbitrary tame supercuspidal representation, even one that does not satisfy the compactness condition; verifying this will be the subject of future work.

2000 Mathematics Subject Classification. Primary 22E50, 22E35.

Key words and phrases. p-adic group, supercuspidal representation, local Langlands correspondence.

The authors were both partially supported by National Science Foundation Focussed-Research Grant 0854897. The second-named author was partially supported by Simons Foundation Collaboration Grant 246066. 
To further explain the content of 4 , we note that the character formulæ of [4] are explicit, in the sense that all ingredients are described in terms of the parametrising data (see Definition 4.1.2); but they can be difficult to evaluate in particular cases. (See, for example, [5, §14] for the case $G=\mathrm{SL}_{2}(k)$.) There are four main ingredients:

- The character of $\pi^{\prime}$. We assume inductively that this is already known. The base case of this induction is provided by depth-0 character formulæ; see [10, Lemma 10.0.4].

- A product of indices of subquotients (see [4, Proposition 5.3.3]). This is discussed in $\$ 4.2$ and computed in Corollary 4.2 .2

- A fourth root of unity (see [4, Propositions 3.8 and 5.2.13]). This is discussed in $\$ 4.3$ and computed (or, at least, re-cast in a form more amenable to stability calculations) in Proposition 4.3.8 Its behaviour is rather subtle; see Proposition 4.3.7 in the unramified case, and [24] for the effects of ramification.

- The Fourier transform of an orbital integral on a centraliser subgroup. This contribution is predicted by Murnaghan-Kirillov theory (see, for example, [15]), and plays the rôle of a Green's function in the Deligne-Lusztig character formulæ (see, for example, [10, Lemma 12.4.3]). We treat it as an uncomputed 'primitive ingredient', although the normalisation of the measure occurring in its definition is quite important. See 4.5 , particularly Proposition 4.5.1.

Finally, in $₫ 5$, we use the character formula Theorem 4.6.2 to show stability of character sums. Here we restrict our attention to toral supercuspidal representations, and, for most of the section (with the exception of Corollary 5.6), even to unramified, toral supercuspidal representations. Motivated by Theorem 4.6.2 we begin by introducing the modified Yutype construction (Definition 5.4) mentioned above. This simplifies the character formula slightly (Corollary 5.6), and allows us to write an analogue (Theorem 5.8) of [10, Lemma 11.0.2] that expresses the sums of the characters in a Reeder-type L-packet in terms of Fourier transforms of stable orbital integrals. Finally, in Theorem 5.10, we use this expression, together with work of Waldspurger on such Fourier transforms ([27, Théorème 1.5] and [10, Lemma 12.2.3]), to prove the desired stability.

\section{NOTATION}

\subsection{Fields and characters.}

Definition 2.1.1. Let $k$ be a field and ord a non-trivial discrete valuation on $k$ (with value group $\mathbb{Z}$ ) with respect to which $k$ is complete. Choose a fixed separable closure $k^{\text {sep }} / k$, and denote the unique extension of ord to $k^{\text {sep }}$ again by ord. Let $k^{\text {un }}$ (respectively, $k^{\text {tame }}$ ) be the maximal unramified (respectively, tame) extension of $k$ inside $k^{\text {sep }}$. Put $\Gamma=\operatorname{Gal}\left(k^{\text {sep }} / k\right)$ and $\Gamma^{\mathrm{un}}=\operatorname{Gal}\left(k^{\mathrm{sep}} / k^{\mathrm{un}}\right)$.

If $E / k$ is a separable extension, then denote by

- $\mathcal{O}_{E}$ the ring of integers of $E$,

- $P_{E}$ the unique maximal ideal in $\mathcal{O}_{E}$,

- $\mathfrak{f}_{E}$ the residue class field $\mathcal{O}_{E} / P_{E}$ of $E$, and

- $q_{E}$ the cardinality of $\mathfrak{f}_{E}$.

We sometimes drop the superscript $E$ when $E=k$. We require that $\mathfrak{f}$ be finite (equivalently, that $k$ be locally compact).

Finally, let Fr be any element of $\Gamma$ whose image in $\operatorname{Gal}\left(\mathfrak{f}_{k^{\text {un }}} / \mathfrak{f}\right)$ is the inverse of $t \mapsto t^{q}$.

Definition 2.1.2. Let $\Lambda$ be an additive character of $k$, i.e., a homomorphism $k \rightarrow \mathbb{C}^{\times}$, chosen as in [4, §1.1]. In particular, $\Lambda$ is trivial on $P$ but not on $\mathcal{O}$. Write $\Lambda$ again for the 
resulting character of $\mathfrak{f}$. Given a finite-dimensional $k$ - or $\mathfrak{f}$-vector space $V$, these characters fix a canonical identification of the linear and Pontrjagin duals of $V$.

Definition 2.1.3. If $V$ is a finite-dimensional $k$ - or $\mathfrak{f}$-vector space, with Pontrjagin dual $\widehat{V}$, and $f: V \rightarrow \mathbb{C}$ is smooth (that is, locally constant with respect to the analytic topology on $V$-which is no condition if the field of scalars is $\mathfrak{f}$ ), then the Fourier transform $\hat{f}: \widehat{V} \rightarrow \mathbb{C}$ of $f$ is defined by

$$
\hat{f}(\chi)=\int_{V} f(X) \chi(X) \mathrm{d} X \quad \text { for all } \chi \in \widehat{V}
$$

where $\mathrm{d} X$ is a suitably normalised Haar measure on $V$ (see below). The choice of additive character $\Lambda$ (Definition 2.1.2) allows us to regard $\hat{f}$ as a function on the linear dual $V^{*}$. Since $\widehat{\hat{V}}$ is canonically identified with $V$, we may, and do, further regard $\hat{\hat{f}}$ as a function on $V$. Then the Haar measure $\mathrm{d} X$ is chosen so that the fourth power of the Fourier transform is the identity.

2.2. Tori and related functions. For the remainder of this paper, let $\mathbf{G}$ be a connected, reductive $k$-group.

Whenever a $k$-group is denoted by a boldface Roman letter, such as $\mathbf{H}$, we denote its Lie algebra by the corresponding boldface Fraktur letter, such as $\mathfrak{h}$. Whenever a $k$ variety is denoted by a boldface letter, such as $\mathbf{Y}$, we denote its set of rational points by the corresponding non-boldface letter, such as $Y$. Thus, for example, $\mathfrak{h}$ is the space of $k$-rational points of the Lie algebra of $\mathbf{H}$. The identity component of $\mathbf{H}$ is denoted by $\mathbf{H}^{\circ}$, and, by abuse of notation, the group of $k$-rational points of $\mathbf{H}^{\circ}$ by $H^{\circ}$ (even though this latter group is actually totally disconnected in the analytic topology inherited from $k$ ).

A maximal torus $\mathbf{T}$ in $\mathbf{G}$ (that is defined over $k$ ) is called a maximal $G$-torus.

Definition 2.2.1. If $\mathbf{T}$ is a maximal $G$-torus, then write $\widetilde{R}(\mathbf{G}, \mathbf{T})$ for the set of weights of the adjoint action of $\mathbf{T}_{k^{\text {sep }}}$ on $\mathfrak{g}_{k^{\text {sep }}}$, and $R(\mathbf{G}, \mathbf{T})=\widetilde{R}(\mathbf{G}, \mathbf{T}) \backslash\{0\}$ for the absolute root system of $\mathbf{T}$ in $\mathbf{G}$.

Definition 2.2.2. An element $\gamma \in G$ (respectively, $X \in \mathfrak{g}$; respectively, $X^{*} \in \mathfrak{g}^{*}$ ) for which the identity component of $C_{\mathbf{G}}(\gamma)$ (respectively, the stabiliser of $X$ in the adjoint action; respectively, the stabiliser of $X^{*}$ in the coadjoint action) is a torus is called regular semisimple. Write $G^{\text {rss }}$ (respectively, $\mathfrak{g}^{\text {rss }}$; respectively, $\mathfrak{g}^{* \text { rss }}$ ) for the set of such elements. A regular, semisimple element with connected centraliser is called strongly regular semisimple [10, §2.9, p. 817].

For the remainder of this section, fix a maximal $G$-torus $\mathbf{T}$, and put $R=R(\mathbf{G}, \mathbf{T})$ and $\widetilde{R}=\widetilde{R}(\mathbf{G}, \mathbf{T})$.

As in [29, $\S 4$, p. 591], we identify the Lie algebra $\mathfrak{t}$ and its dual $\mathfrak{t}^{*}$ with the subgroups of $\mathfrak{g}$ and $\mathfrak{g}^{*}$, respectively, fixed by the (adjoint or coadjoint, respectively) action of $T$.

The terminology and symbols below (Definitions 2.2.3 and 2.2.4) make sense for arbitrary characters, but we need them only for roots. The notation is as in [4, Notation 3.6], except that we write $k_{\alpha}$ in place of $F_{\alpha}$, and $\sigma_{\alpha}$ in place of $\eta_{\alpha}$.

Definition 2.2.3. If $\alpha \in R$, then $\alpha$ is symmetric exactly when its $\Gamma$-orbit contains $-\alpha$ (and non-symmetric otherwise). A symmetric root is ramified exactly when its $\Gamma^{\mathrm{un}}$-orbit contains $-\alpha$ (and unramified otherwise).

If $S$ is any subset of $R$, then $S_{\text {symm }}, S_{\text {symm, unram }}, S_{\text {symm, ram }}$, and $S^{\text {symm }}$ are the sets of roots in $S$ that are symmetric, symmetric and unramified, symmetric and ramified, and non-symmetric, respectively. If $\alpha \in R_{\text {symm }}$, then let $\sigma_{\alpha}$ be any element of $\Gamma^{\text {un }}$ (if $\alpha$ is ramified) or of $\Gamma$ (if $\alpha$ is unramified) such that $\sigma_{\alpha} \alpha=-\alpha$. 
Definition 2.2.4. If $\alpha \in \widetilde{R}$, then put $\Gamma_{\alpha}=\operatorname{stab}_{\Gamma} \alpha$ and $\Gamma_{ \pm \alpha}=\operatorname{stab}_{\Gamma}\{ \pm \alpha\}=\left\langle\Gamma_{\alpha}, \sigma_{\alpha}\right\rangle$. Write $k_{\alpha}$ for the fixed field in $k^{\text {sep }}$ of $\Gamma_{\alpha}$, and similarly for $k_{ \pm \alpha}$. All our notations below with subscript $\alpha$ have obvious analogues with subscript $\pm \alpha$, which we use as necessary. Write

- $\mathcal{O}_{\alpha}=\mathcal{O}_{k_{\alpha}}$ and $P_{\alpha}=P_{k_{\alpha}}$;

- $n_{\alpha}, e_{\alpha}$, and $f_{\alpha}$ for the degree, ramification degree, and residual degree, respectively, of $k_{\alpha} / k$;

- $\mathfrak{f}_{\alpha}=\mathfrak{f}_{k_{\alpha}}$, and $q_{\alpha}=q_{k_{\alpha}}$;

- if $\alpha$ is symmetric and unramified, then $\mathfrak{f}_{\alpha}^{1}$ is the kernel of the norm map from $\mathfrak{f}_{\alpha}$ to $\mathfrak{f}_{ \pm \alpha} ;$ and

- $\mathbb{Z}_{\alpha}=\operatorname{ord}\left(k_{\alpha}^{\times}\right)=e_{\alpha}^{-1} \mathbb{Z}$ for the value group of $k_{\alpha}$.

These objects depend only on the $\Gamma$-orbit $\omega$ of $\alpha$; so we denote them instead by $k_{\omega}, \&$ c., when convenient.

Definition 2.2.5. If $\alpha \in \widetilde{R}$, then $\mathfrak{g}_{\alpha}$ is the $\alpha$-weight space for the action of $\mathbf{T}_{k_{\alpha}}$ on $\mathfrak{g}_{k_{\alpha}}$, and $\mathbf{G}_{\alpha}$ is the corresponding subgroup of $\mathbf{G}_{k_{\alpha}}$ (denoted by $U_{\alpha}$ in [25, Proposition 8.1.1(i)] when $\alpha \neq 0$ ). Note that these are defined only over $k_{\alpha}$, not necessarily over $k$.

Remark 2.2.6. In particular, $\mathfrak{g}_{0}=\mathfrak{t}$ and $\mathbf{G}_{0}=\mathbf{T}$, both of which are defined over $k_{0}=k$.

Definition 2.2.7. For $\gamma \in T$ and $X \in \mathfrak{t}$, and any character $\chi$ of $\mathbf{T}_{k^{\mathrm{sep}}}$, put

$$
\operatorname{ord}_{\gamma} \chi=\operatorname{ord}(\chi(\gamma)-1) \quad \text { and } \operatorname{ord}_{X} \chi=\operatorname{ord} \chi(X) .
$$

For $X^{*} \in \mathfrak{t}^{*}$ and $\alpha \in R$, define $\mathrm{d} \alpha^{\vee}\left(X^{*}\right):=X^{*}\left(\mathrm{~d} \alpha^{\vee}(1)\right)$ and put $\operatorname{ord}_{X^{*}} \alpha=\operatorname{ord} \mathrm{d} \alpha^{\vee}\left(X^{*}\right)$.

Note that $\operatorname{ord}_{\gamma}$ is a non-negative function exactly when $\gamma$ is bounded (that is, has bounded orbits on the set $\mathcal{B}(\mathbf{G}, k)$ of Definition 3.0.2 below, in the sense of [7, Exemple 3.1.2(b)]).

It is well known that the discriminant function on $G$ (see, for example, [13, §VI.1, p. 63]) controls the 'blow up' of characters [13, Theorem VI.8.14]. We introduce a slight variant where, rather than taking the globally lowest coefficient in a characteristic polynomial (which may vanish), we take the pointwise lowest coefficient (which never vanishes). It seems that this may actually provide a better measure of the blow up of the character; at least, it gives us a more natural inductive formula for characters (Theorem 4.6.2).

Definition 2.2.8. If $\gamma \in G$ (respectively, $X \in \mathfrak{g}$ ), then $D_{G}^{\text {red }}(\gamma)$ (respectively, $D_{G}^{\text {red }}(X)$ ) is the coefficient of the lowest-degree power of $t$ in $\operatorname{det}(\operatorname{Ad}(\gamma)-1+t)$ (respectively, $\operatorname{det}(\operatorname{ad}(X)+t))$.

Remark 2.2.9. Preserve the notation of Definition 2.2.8. If $\gamma \in G$ and $X \in \mathfrak{g}$ have Jordan decompositions $\gamma=\gamma_{\mathrm{ss}} \cdot \gamma_{\mathrm{un}}$ and $X=X_{\mathrm{ss}}+X_{\text {nil }}$, respectively, then $D_{G}^{\mathrm{red}}(\gamma)=D_{G}^{\mathrm{red}}\left(\gamma_{\mathrm{ss}}\right)$ and $D_{G}^{\mathrm{red}}(X)=D_{G}^{\mathrm{red}}\left(X_{\mathrm{ss}}\right)$. Now suppose that $\gamma \in T$ and $X \in \mathfrak{t}$. By passing to a splitting field for $\mathbf{T}$, we see that

$$
D_{G}^{\mathrm{red}}(\gamma)=\prod_{\substack{\alpha \in R \\ \alpha(\gamma) \neq 1}}(\alpha(\gamma)-1) \quad \text { and } \quad D_{G}^{\mathrm{red}}(X)=\prod_{\substack{\alpha \in R \\ \mathrm{~d} \alpha(X) \neq 0}} \mathrm{~d} \alpha(X),
$$

hence that

$$
\left|D_{G}^{\mathrm{red}}(\gamma)\right|=\prod_{\substack{\alpha \in R \\ \alpha(\gamma) \neq 1}} q^{-\operatorname{ord} \alpha} \text { and }\left|D_{G}^{\mathrm{red}}(X)\right|=\prod_{\substack{\alpha \in R \\ \mathrm{~d} \alpha(X) \neq 0}} q^{-\operatorname{ord}_{X} \alpha} .
$$


It is therefore natural to define

$$
D_{G}^{\mathrm{red}}\left(X^{*}\right)=\prod_{\substack{\alpha \in R \\ \mathrm{~d} \alpha^{\vee}\left(X^{*}\right) \neq 0}} \mathrm{~d} \alpha^{\vee}\left(X^{*}\right) \quad \text { for all } X^{*} \in \mathfrak{t}^{*} .
$$

\section{MOY-PRASAD FILTRATIONS}

As in 2.2 let $\mathbf{G}$ be a connected, reductive $k$-group.

Definition 3.0.1. Put $\widetilde{\mathbb{R}}=\mathbb{R} \sqcup\{r+\mid r \in \mathbb{R}\}$. This carries a natural order, and an orderreversing involution $\widetilde{r}$ defined by $\tilde{r}=(-r)+$ and $\widetilde{r+}=-r$ for $r \in \mathbb{R}$.

Note that, unlike [3, §3], we do not find it convenient to include $+\infty$ in $\widetilde{\mathbb{R}}$.

Definition 3.0.2. Write $\mathcal{B}(\mathbf{G}, k)$ for the enlarged, and $\mathcal{B}^{\text {red }}(\mathbf{G}, k)$ for the reduced, BruhatTits building of $\mathbf{G}$ over $k$ ([7, Définition 7.4.2] and [8, §4.2.16]). There is a natural projection $\mathcal{B}(\mathbf{G}, k) \rightarrow \mathcal{B}^{\text {red }}(\mathbf{G}, k)$, denoted by $x \mapsto \bar{x}$.

Remark 3.0.3. If $\mathbf{T}$ is a $k^{\text {tame }}$-split, maximal $G$-torus, then there is a family of embeddings of $\mathcal{B}(\mathbf{T}, k)$ in $\mathcal{B}(\mathbf{G}, k)$, with canonical image. (To see this, regard the apartment $\mathcal{A}\left(\mathbf{T}_{E}\right)$ of $\mathbf{T}_{E}$ as a subsimplex of $\mathcal{B}(\mathbf{G}, E)$, where $E / k$ is the splitting field of $\mathbf{T}$, and then realise $\mathcal{B}(\mathbf{T}, k)$ and $\mathcal{B}(\mathbf{G}, k)$ as the sets of $\Gamma$-fixed points of $\mathcal{A}\left(\mathbf{T}_{E}\right)$ and $\mathcal{B}(\mathbf{G}, E)[26$, 2.6 .1$]$.)

For the remainder of this section, fix a $k^{\text {tame }}$-split, maximal $G$-torus $\mathbf{T}$ and a point $x \in \mathcal{B}(\mathbf{G}, k)$ lying in the image of $\mathcal{B}(\mathbf{T}, k)$ (which we abbreviate to just " $x \in \mathcal{B}(\mathbf{T}, k)$ "). Put $R=R(\mathbf{G}, \mathbf{T})$ and $\widetilde{R}=\widetilde{R}(\mathbf{G}, \mathbf{T})$.

\subsection{Valuations of root data.}

Definition 3.1.1. If $r \in \widetilde{\mathbb{R}}$, then write

$$
\mathfrak{g}_{x, r}, \quad \mathfrak{g}_{x, r}^{*}, \quad \text { and } \quad G_{x, r}(\text { if } r \geq 0)
$$

for the associated Moy-Prasad filtration subgroups of $\mathfrak{g}, \mathfrak{g}^{*}$, and $G$, respectively (see [19, $\S \S 2.6,3.2,3.5]$ and [20, $\S \S 3.2,3.3])$. Also, put $G_{r}=\bigcup_{y \in \mathcal{B}(\mathbf{G}, k)} G_{y, r}$ and $\mathfrak{g}_{r}=$ $\bigcup_{y \in \mathcal{B}(\mathbf{G}, k)} \mathfrak{g}_{y, r}$.

In particular, $G_{x, 0}$ is the parahoric subgroup of $G$ associated to $x$ [8, Définition 5.2.6], and $\mathfrak{g}_{x, r}^{*}=\left\{X^{*} \in \mathfrak{g}^{*} \mid\left\langle X^{*}, Y\right\rangle \in P\right.$ for all $\left.Y \in \mathfrak{g}_{x, \tilde{r}}\right\}$.

If $\mathcal{G}$ is a group equipped with a filtration $\left(\mathcal{G}_{i}\right)_{i \in I}$ by subgroups, then we frequently write $\mathcal{G}_{i: j}$ in place of $\mathcal{G}_{i} / \mathcal{G}_{j}$ when $\mathcal{G}_{j} \subseteq \mathcal{G}_{i}$ (even if the quotient is not a group). Thus, for example, $G_{x, 0: 0+}$ is the group of $\mathfrak{f}$-points of a reductive $\mathfrak{f}$-group [19, §3.2, p. 398].

Our (rational) character computation Theorem 4.6.2 requires the existence of a 'mockexponential' map that behaves, in many ways, like an exponential map, but may sacrifice some equivariance in order to converge on a larger domain. Hypothesis 3.1.2 below, which depends implicitly on a positive rational number $r$, formalises this. Note, however, that we do not need this hypothesis until Theorem 4.6.2 (at which point $r$ stands for the depth of the representation whose character we compute there). We also require the hypothesis for the consequences of Theorem 4.6.2 in $₫ 5$, but, in that section, we actually impose the stronger hypothesis [10, Restriction 12.4.1(2)].

Hypothesis 3.1.2. There exists a family of bijections

$$
\begin{array}{r}
\left(\mathrm{e}_{\mathbf{S}, \bar{y}}: \mathfrak{g}_{y, r} \rightarrow G_{y, r}\right)_{\mathbf{S} a k^{\text {tame }}-\text { split maximal G-torus }} \\
\qquad \bar{y} \in \operatorname{im} \mathcal{B}(\mathbf{S}, k) \rightarrow \mathcal{B}^{\text {red }}(\mathbf{G}, k)
\end{array}
$$

satisfying [4, Hypotheses A.1 and A.7], and, whenever 
- $\mathbf{H}$ is a reductive subgroup of $\mathbf{G}$,

- $\mathbf{S}$ is a $k^{\text {tame }}$-split maximal $G$-torus that is contained in $\mathbf{H}$,

- $y \in \mathcal{B}(\mathbf{S}, k)$, and

- $Y \in \mathfrak{h}_{x, r}$,

also the following conditions:

(1) $\left|D_{H}^{\mathrm{red}}(Y)\right|=\left|D_{H}^{\mathrm{red}}\left(\mathrm{e}_{\mathbf{S}, \bar{y}} Y\right)\right|$, and

(2) if $g \in G$ and $\operatorname{Int}(g) \mathrm{e}_{\mathbf{S}, \bar{y}} Y \in G_{y, r}$, then

$$
\hat{\mu}_{\mathrm{Ad}^{*}(g) X^{*}}^{\operatorname{Int}(g) H}\left(\mathrm{e}_{\mathbf{S}, \bar{x}}^{-1} \operatorname{Int}(g) \mathrm{e}_{\mathbf{S}, \bar{x}} Y\right)=\hat{\mu}_{X^{*}}^{H}(\operatorname{Ad}(g) Y) \quad \text { for all } X^{*} \in \mathfrak{h}_{x,-r}^{*},
$$

where $\hat{\mu}$ is as in Definition 4.1.6

The point $x \in \mathcal{B}(\mathbf{T}, k)$ defines a 'valuation of root datum', roughly in the sense of [7, §6.1.1], except that we must take into account the fact that $\mathbf{T}$ need not be $k$-split (nor even maximally $k$-split).

Definition 3.1.3. For $\alpha \in \widetilde{R}$ and $i \in \widetilde{\mathbb{R}}$, write $\mathfrak{g}_{\alpha}\left(k_{\alpha}\right)_{x, i}:=\mathfrak{g}_{\alpha}\left(k_{\alpha}\right) \cap \mathfrak{g}\left(k_{\alpha}\right)_{x, i}$, and put $\operatorname{ord}_{x} \alpha=\left\{i \in \mathbb{R} \mid \mathfrak{g}_{\alpha}\left(k_{\alpha}\right)_{x, i: i+} \neq 0\right\}$.

Remark 3.1.4. Note that $\operatorname{ord}_{x} \alpha$ implicitly depends on the underlying field $k$ (and on the normalisation of its valuation). Since $k_{\alpha}^{\text {un }} / k_{\alpha}$ is unramified, we have that $\mathfrak{g}\left(k_{\alpha}\right)_{x, i: i+}=$ $\mathfrak{g}\left(k_{\alpha}^{\text {un }}\right)_{x, i: i+}^{\Gamma_{\alpha}}$ [29, Corollary 2.3], hence, by Hilbert's Theorem 90 [22, Proposition II.1.2.1], that $\mathfrak{g}\left(k_{\alpha}\right)_{x, i: i+} \neq 0$ if and only if $\mathfrak{g}\left(k_{\alpha}^{\text {un }}\right)_{x, i: i+} \neq 0$. In other words, $\operatorname{ord}_{x} \alpha$ does not change if $k$ is replaced by an unramified extension.

Remark 3.1.5. Since $\mathfrak{g}_{\alpha}\left(k_{\alpha}\right)$ is a 1 -dimensional $k_{\alpha}$-space, we have that $\operatorname{ord}_{x} \alpha$ is a $\mathbb{Z}_{\alpha^{-}}$ torsor. With the terminology of [19, §2.5],

$$
\operatorname{ord}_{x} \alpha=\left\{\psi(x) \mid \psi \text { is an affine } k_{\alpha} \text {-root with gradient } \alpha\right\} \text {. }
$$

Lemma 3.1.6 below is of interest mainly because of its consequences, Corollaries 3.1 .8 and 3.1 .9

Lemma 3.1.6. If $\alpha \in R$ and $i, j \in \widetilde{\mathbb{R}}$ with $i \leq j$, then the pairing

$$
\mathfrak{g}_{\alpha}\left(k_{\alpha}\right)_{x, i: j} \otimes \mathfrak{g}_{-\alpha}\left(k_{\alpha}\right)_{x, \tilde{\jmath}: \tilde{\imath}} \stackrel{[\cdot, \cdot]}{\longrightarrow} \mathfrak{t}\left(k_{\alpha}\right)_{0: 0+} \stackrel{\alpha}{\rightarrow} \mathfrak{f}_{\alpha}
$$

is non-degenerate.

Proof. Let $\mathbf{G}_{ \pm \alpha}$ be the $k_{\alpha}$-split, rank-1, semisimple Lie group generated by the ( $\left.\pm \alpha\right)$-root subgroups of G. By [25, §16.3.1], the proof of [25, Theorem 7.2.4] goes through to show that there is a $k_{\alpha}$-surjection $\mathbf{G}_{ \pm \alpha} \rightarrow \mathrm{PGL}_{2}$. (Alternatively, a more machinery-heavy proof could apply the isomorphism theorem [25, Theorem 16.3.2] to the obvious morphism from the root datum of $\mathrm{PGL}_{2}$ to that of $\mathbf{G}_{ \pm \alpha}$.) A p $\mathfrak{p l}_{2}$-calculation then establishes the result.

Corollary 3.1.7. With the notation of Lemma 3.1.6 there is a non-canonical $\mathcal{O}_{\alpha}$-module isomorphism of $\mathfrak{g}_{\alpha}\left(k_{\alpha}\right)_{x, i: j}$ with $\mathfrak{g}_{-\alpha}\left(k_{\alpha}\right)_{x, \tilde{\jmath}: \tau}$.

Proof. This follows from Lemma 3.1.6 and the fact that a choice of $k_{\alpha}$-basis of $\mathfrak{g}_{\alpha}\left(k_{\alpha}\right)$ furnishes an isomorphism of $\mathfrak{g}_{\alpha}\left(k_{\alpha}\right)_{x, i: j}$ with a quotient of fractional ideals of $\mathcal{O}_{\alpha}$. Such a quotient is self-dual (as an $\mathcal{O}_{\alpha}$-module).

Corollary 3.1.8. For any $\alpha \in R$, we have that $\operatorname{ord}_{x}(-\alpha)=-\operatorname{ord}_{x}(\alpha)$.

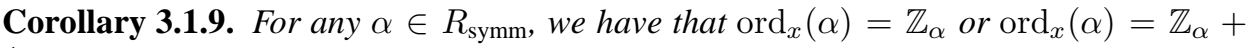
$\frac{1}{2} e_{\alpha}^{-1}$. 
3.2. Subgroups associated to functions on root systems. Most of this section is devoted establishing analogues of the results of [3, §5.2] for subgroups of $\mathfrak{g}$ attached to functions on root systems. The proofs here are considerably easier, because the group structure of $\mathfrak{g}$ is much simpler than that of $G$, and because Galois descent behaves better for algebras than groups, so that we get to ignore many technical complications. For example, we do not have to restrict our attention to concave functions [3, Definition 5.7], or intersect with a parahoric subalgebra as in [3, Definition 5.14].

Definition 3.2.1. If $f: \Gamma \backslash \widetilde{R} \rightarrow \widetilde{\mathbb{R}} \cup\{+\infty\}$ is any function (not necessarily concave), then put

$$
\mathbf{T} \mathfrak{g}_{x, f}=\left(\bigoplus_{\substack{\alpha \in \widetilde{R} \\ f(\alpha)<+\infty}} \mathfrak{g}_{\alpha}\left(k_{\alpha}\right)_{x, f(\alpha)}\right)^{\Gamma},
$$

with the notation of Definition 3.1.3 (See also [4, §3.2, p. 1144].)

The group algebra $\mathcal{O}[T]$ of the torus $T$ acts naturally on $\mathfrak{g}$, on $\mathfrak{g}_{\alpha}$, and on each ${ }_{T} \mathfrak{g}_{x, f}$ (via their natural $\mathcal{O}$-module structures, and the adjoint action of $T$ ). We prove a few basic results about its action on groups of the form ${ }_{\mathbf{T}} \mathfrak{g}_{x, f}$ that will be needed in the proof of Lemma 3.2.5

Lemma 3.2.2. There is a canonical $\mathcal{O}[T]$-module isomorphism

$$
\mathfrak{g} \cong \bigoplus_{\alpha \in \Gamma \backslash \widetilde{R}} \mathfrak{g}_{\alpha}\left(k_{\alpha}\right)
$$

that restricts, for any function $f: \Gamma \backslash \widetilde{R} \rightarrow \widetilde{\mathbb{R}} \cup\{+\infty\}$, to an isomorphism

$$
\mathbf{T} \mathfrak{g}_{x, f} \cong \bigoplus_{\alpha \in \Gamma \backslash \widetilde{R}} \mathfrak{g}_{\alpha}\left(k_{\alpha}\right)_{x, f(\alpha)} .
$$

Proof. The inverse isomorphism sends $\left(X_{\alpha}\right)_{\alpha \in \Gamma \backslash \widetilde{R}}$ to $\sum_{\alpha \in \Gamma \backslash \widetilde{R}} \sum_{\sigma \in \Gamma / \Gamma_{\alpha}} \sigma X_{\alpha}$. It is straightforward to check that its restriction has the desired properties.

Corollary 3.2.3. Suppose that $f, g: \Gamma \backslash \widetilde{R} \rightarrow \widetilde{\mathbb{R}} \cup\{+\infty\}$, with

- $f(0)=g(0)$,

- $f(\alpha) \leq g(\alpha)$ for all $\alpha \in \widetilde{R}$, and

- $g(\alpha)<+\infty$ whenever $f(\alpha)<+\infty$.

Then there is a non-canonical $\mathcal{O}$-module isomorphism ${ }_{\mathbf{T}} \mathfrak{g}_{x, f: g} \cong{ }_{\mathbf{T}} \mathfrak{g}_{x, \tilde{g}: \tilde{f}}$, where

$$
\tilde{f}(\alpha)= \begin{cases}\widetilde{f(-\alpha)}, & f(-\alpha)<+\infty \\ +\infty, & \text { otherwise, }\end{cases}
$$

and similarly for $\tilde{g}$.

Proof. By Corollary 3.1.7, there is a non-canonical $\mathcal{O}$-module isomorphism

$$
\bigoplus_{\alpha \in \Gamma \backslash \widetilde{R}} \mathfrak{g}_{\alpha}\left(k_{\alpha}\right)_{x, f(\alpha): g(\alpha)} \cong \bigoplus_{\alpha \in \Gamma \backslash \widetilde{R}} \mathfrak{g}_{-\alpha}\left(k_{\alpha}\right)_{x, \tilde{g}(-\alpha): \tilde{f}(-\alpha)},
$$

which, by Lemma 3.2.2 may be transferred to the desired isomorphism. 
Lemma 3.2.4. If $\left(r_{\alpha}\right)_{\alpha \in \Gamma \backslash R}$ is a collection of rational numbers such that $r_{\alpha} \in \mathbb{Z}_{\alpha}$ for all $\alpha \in R$, then there is a non-canonical $\mathcal{O}[T]$-module automorphism of $\mathfrak{g}$ that restricts, for any function $f: \Gamma \backslash \widetilde{R} \rightarrow \widetilde{\mathbb{R}} \cup\{+\infty\}$, to an isomorphism

$$
\mathbf{T} \mathfrak{g}_{x, f} \cong \mathbf{T} \mathfrak{g}_{x, f+r}
$$

(where $(f+r)(0):=f(0)$ and $(f+r)(\alpha):=f(\alpha)+r_{\alpha}$ for all $\left.\alpha \in R\right)$, and that has determinant there of valuation $\sum_{\substack{\alpha \in R \\ f(\alpha)<+\infty}} r_{\alpha}$.

Proof. By hypothesis, there is a collection $\left(c_{\alpha}\right)_{\alpha \in \Gamma \backslash R}$ such that $c_{\alpha} \in k_{\alpha}$ and $\operatorname{ord}\left(c_{\alpha}\right)=r_{\alpha}$ for all $\alpha \in R$. By Lemma 3.2.2, the automorphism of $\bigoplus_{\alpha \in \Gamma \backslash \widetilde{R}} \mathfrak{g}_{\alpha}\left(k_{\alpha}\right)$ that is the identity on the $\alpha=0$ summand, and that multiplies the summand corresponding to $\alpha \in R$ by $c_{\alpha}$, may be transferred to the desired automorphism of $\mathfrak{g}$.

Lemma 3.2.5 is crucial in the calculation of Proposition 4.2.1, where it accounts for the appearance in the character formula Theorem 4.6.2 of a blow-up controlled by the discriminant.

Lemma 3.2.5. Suppose that $f, g: \Gamma \backslash \widetilde{R} \rightarrow \mathbb{R} \cup\{+\infty\}$ are functions such that

- $f(\alpha) \leq g(\alpha)$ for all $\alpha \in \widetilde{R}$,

- $f(\alpha)<+\infty$ whenever $f(-\alpha)<+\infty$, and similarly for $g(\alpha)$,

- $g(\alpha)<+\infty$ whenever $f(\alpha)<+\infty$, and

- $f(\alpha)+f(-\alpha), g(\alpha)+g(-\alpha) \in \mathbb{Z}_{\alpha} \cup\{+\infty\}$ for all $\alpha \in R$.

Then

$$
\begin{aligned}
\left|\mathfrak{T}_{x, f: g}\right| \cdot\left|\mathbf{T}_{x, f+: g+}\right| & \\
& =\left|\mathfrak{t}_{f(0): g(0)}\right|\left|\mathfrak{t}_{f(0)+: g(0)+}\right| \cdot \prod_{\substack{\alpha \in R \\
f(\alpha)<+\infty}} q^{(g(\alpha)+g(-\alpha))-(f(\alpha)+f(-\alpha)) .}
\end{aligned}
$$

Remark 3.2.6. In our application of Lemma 3.2.5 (see the proof of Proposition 4.2.1), we have that $f(0)=g(0)$ and that $f$ and $g$ are $\pm \Gamma$-, not just $\Gamma$-, invariant; so then the condition on their values becomes that $f(\alpha), g(\alpha) \in \frac{1}{2} \mathbb{Z}_{\alpha}$ for all $\alpha \in R$, and the cardinality becomes

$$
\prod_{\substack{\alpha \in\langle \pm 1\rangle \backslash R \\ f(\alpha)<+\infty}} q^{2(g(\alpha)-f(\alpha))} .
$$

Proof. We have that $\mathbf{T} \mathfrak{g}_{x, f: g}=\mathfrak{t}_{x, f(0): g(0)} \oplus \mathfrak{g}_{x, f_{R}: g_{R}}$, where $f_{R}$ and $g_{R}$ agree with $f$ and

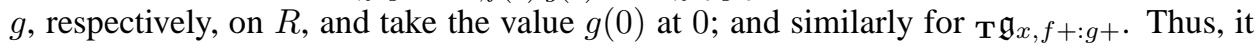
suffices to consider the case where $f(0)=g(0)$. In this case, put $r_{\alpha}=0$ if $f(\alpha)=+\infty$, and

$$
r_{\alpha}=g(\alpha)-(\widetilde{g+})(\alpha)=g(\alpha)+g(-\alpha) \in \mathbb{Z}_{\alpha}
$$

otherwise. By Corollary 3.2 .3 and Lemma 3.2.4 we have that

$$
\mathbf{T} \mathfrak{g}_{x, f+: g+} \cong \mathbf{T}_{x, \widetilde{g+}: \widetilde{f+}} \cong \mathbf{T}_{x,((\widetilde{g+})+r):((\widetilde{f+})+r)}=\mathbf{T}_{x, g:((\widetilde{f+})+r)},
$$

hence that

$$
\left|\mathfrak{T}_{x, f: g}\right| \cdot\left|{ }_{\mathbf{T}} \mathfrak{g}_{x, f+: g+}\right|=\left|{ }_{\mathbf{T}} \mathfrak{g}_{x, f: g}\right| \cdot\left|{ }_{\mathbf{T}} \mathfrak{g}_{x, g:((\widetilde{f+})+r)}\right|=\left|{ }_{\mathbf{T}} \mathfrak{g}_{x, f:((\widetilde{f+})+r)}\right| .
$$

Now, whenever $f(\alpha)<+\infty$, we have that

$$
s_{\alpha}:=((\widetilde{f+})+r)(\alpha)-f(\alpha)=(g(\alpha)+g(-\alpha))-(f(\alpha)+f(-\alpha))
$$


lies in $\mathbb{Z}_{\alpha}$, so, by Lemma 3.2.4 again,

$$
\left|\mathbf{T g}_{x, f:((\widetilde{f+})+r)}\right|=\left|\operatorname{det}\left(\mathbf{T}_{\mathfrak{g}_{x, f}} \rightarrow \mathbf{T} \mathfrak{g}_{x,((\widetilde{f+})+r)}\right)\right|^{-1}=\prod_{\alpha \in R} q^{s_{\alpha}} .
$$

\section{Character Formul}

As in $\sqrt[3]{3}$, let $\mathbf{G}$ be a connected, reductive $k$-group. This section is devoted to making more explicit the computations of [4] of the characters of supercuspidal representations of $G$. To use those computations, we must assume that $\mathbf{G}$ satisfies [3, Hypotheses B and C], so we do so for the remainder of the paper. In order that the theory not be vacuous, we also assume for the remainder of the paper that $\mathbf{G}$ possesses a $k^{\text {tame }}$-split, maximal torus, so that $\mathbf{G}$ also satisfies [3, Hypotheses A and D] (see [3, Remark 2.2]).

We begin by recalling Yu's construction [29] of the so called 'tame' supercuspidal representations of $G$; the necessary notation is introduced in 4.1 . The next four subsections handle the ingredients of the character formulæ of [4], as described in the introduction. Finally, in 4.6 , we tie together these results to give an inductive formula (Theorem 4.6.2) that computes the character of a tame supercuspidal representation in terms of the character of a supercuspidal representation of a smaller group, together with what we believe to be a minimal collection of auxiliary data (the fourth roots of unity occurring in Definition 4.3.1.

4.1. Notation. We begin by defining, inductively (on the semisimple rank of $\mathbf{G}$, say), a parametrising set for the supercuspidal representations that we consider.

Definition 4.1.1. A cuspidal $G$-pair is a pair $\left(\mathbf{G}^{\prime}, \pi^{\prime}\right)$, where

- $\mathbf{G}^{\prime}$ is a $\left(k\right.$-)subgroup of $\mathbf{G}$ such that $Z\left(\mathbf{G}^{\prime}\right) / Z(\mathbf{G})$ is $(k$-)anisotropic,

- $\mathbf{G}_{k^{\text {tame }}}^{\prime}$ is a Levi subgroup of $\mathbf{G}_{k^{\text {tame }}}$,

- $\pi^{\prime}$ is a tame supercuspidal representation of $G^{\prime}$, and

- there is a character $\phi$ of $G^{\prime}$ such that the depth [19, Theorem 5.2] of $\pi^{\prime} \otimes \phi^{-1}$ is strictly less than that of $\pi^{\prime}$.

The depth of the cuspidal pair is the depth of $\pi^{\prime}$. (Recall that a tame supercuspidal representation is one that arises via Yu's construction [29, §4] from a datum involving generic characters. See Definition 4.1.2.)

The pair is toral if $\mathbf{G}^{\prime}$ is a torus, and compact if $\mathbf{G}^{\prime} / Z(\mathbf{G})$ is $k$-anisotropic. We sometimes write $\left(G^{\prime}, \pi^{\prime}\right)$ instead of $\left(\mathbf{G}^{\prime}, \pi^{\prime}\right)$. (Since $G^{\prime}$ is Zariski-dense in $\mathbf{G}^{\prime}$, 6, Corollary 18.3], this notational convenience causes no ambiguity.)

The somewhat artificial condition on depth in Definition 4.1.1 ensures that the character $\phi_{d-1}$ of Definition 4.1.2 is not trivial, hence that the 'induction' $\mathrm{Y}_{G^{\prime}}^{G} \pi^{\prime}$ defined there makes sense.

Definition 4.1.2. By the definition of a tame supercuspidal representation, if $\left(\mathbf{G}^{\prime}, \pi^{\prime}\right)$ is a depth- $r$, cuspidal $G$-pair, then $\pi^{\prime}$ is associated to a (non-unique) generic $G^{\prime}$-datum

$$
\left(\left(\mathbf{G}^{0} \subsetneq \cdots \subsetneq \mathbf{G}^{d-1}=\mathbf{G}^{\prime}\right), x,\left(r_{0}, \ldots, r_{d-1}\right),\left(\phi_{0}, \ldots, \phi_{d-1}\right), \rho\right),
$$

in the sense of [16, §0.3] (see also [29, §3]). In particular, $r_{d-1}=r$; and, by Definition 4.1.1, $\phi_{d-1} \neq 1$. Then $\left(G^{\prime}, \pi^{\prime}, \phi_{d-1}, x, X^{*}\right)$ is called an expanded cuspidal G-quintuple corresponding to $\left(G^{\prime}, \pi^{\prime}\right)$ exactly when $X^{*}$ is $\left(\left(\mathbf{G}^{\prime}, \mathbf{G}\right)\right.$-) generic, in the sense of [29, $\S 8$, p. 596], and realises $\phi_{d-1}$ on $G_{x, r}^{\prime}$ (relative to $x$ ), in the sense of [29, §5, p. 593]. By definition, every cuspidal $G$-pair has a corresponding expanded cuspidal $G$-quintuple. 
Write $\mathrm{Y}_{G^{\prime}}^{G} \pi^{\prime}$ for the supercuspidal representation of $G$ constructed by $\mathrm{Yu}$ [29, §4] from the generic $G$-datum

$$
\left(\left(\mathbf{G}^{0} \subsetneq \cdots \subsetneq \mathbf{G}^{d-1}=\mathbf{G}^{\prime} \subsetneq \mathbf{G}^{d}=\mathbf{G}\right), x,\left(r_{0}, \ldots, r_{d-1}, r_{d-1}\right),\left(\phi_{0}, \ldots, \phi_{d-1}, 1\right), \rho\right) .
$$

By [12, Theorem 6.6], under a centrality condition analogous to our Definition 4.1.4 below [12, Hypothesis $\mathrm{C}(\mathbf{G})$ and Remark 2.49], the representation $\mathrm{Y}_{G^{\prime}}^{G} \pi^{\prime}$ depends only on $\pi^{\prime}$, not on the choice of generic $G$-datum; but this deep fact is used here only as a notational convenience.

Remark 4.1.3. If $\left(G^{\prime}, \pi^{\prime}, \phi, x, X^{*}\right)$ and $\left(G^{\prime}, \pi^{\prime}, \phi^{\prime}, x^{\prime}, X^{\prime *}\right)$ are depth-r, expanded cuspidal $G$-quintuples corresponding to the same cuspidal $G$-pair $\left(G^{\prime}, \pi^{\prime}\right)$, then, by [29, Lemma $3.3]$, the images of $x$ and $x^{\prime}$ in the reduced building $\mathcal{B}^{\text {red }}(\mathbf{G}, k)$ are in the same $G^{\prime}$-orbit. By construction [29, §4], since $\pi^{\prime}$ is the twist by $\phi$ of a representation of depth strictly less than $r$, we have that $\left.\pi^{\prime}\right|_{G_{x, r}^{\prime}}$ is $\phi$-isotypic, and similarly for $\phi^{\prime}$. Thus, $\phi$ and $\phi^{\prime}$ agree on (the $G^{\prime}$-orbit of) $G_{x, r}^{\prime}$, so that $X^{\prime *} \in X^{*}+\mathfrak{z}\left(\mathfrak{g}^{\prime}\right)_{(-r)+}^{*}$. We 'rigidify' $X^{*}$ further in Definition 4.1.4 below.

The next definition builds in [4, Hypothesis 2.3] to our definition of a cuspidal pair.

Definition 4.1.4. In the notation of Definition 4.1.2 the depth- $r$, expanded cuspidal $G$ quintuple $\left(G^{\prime}, \pi^{\prime}, \phi, x, X^{*}\right)$ is central exactly when $X^{*}$ realises $\phi$ on $G_{x,(r / 2)+}^{\prime}$ (not just on $G_{x, r}^{\prime}$ ), in the sense of [29, $\S 5$, p. 593]; and a cuspidal $G$-pair is central if it has a corresponding central, expanded cuspidal $G$-quintuple.

Note that a toral, cuspidal $G$-pair is automatically compact and central.

Remark 4.1.5. In the notation of Remark 4.1.3, if the expanded cuspidal quintuples occurring there are central, then $X^{\prime *} \in X^{*}+\mathfrak{z}\left(\mathfrak{g}^{\prime}\right)_{x,-r / 2}^{*}$.

We now introduce notation for the basic objects of harmonic analysis, namely, characters and (Fourier transforms of) orbital integrals. We work with characters and orbital integrals that have been 'normalised' by multiplying by the square root of the (reduced) discriminant to remove singularities.

Definition 4.1.6. Recall that, if $\pi$ is a smooth irreducible representation of $G$ and $X^{*}$ is a semisimple element of $\mathfrak{g}^{*}$ (i.e., if it is fixed by the co-adjoint action of some maximal torus of $G$ ), then the distribution character

$$
F \mapsto \operatorname{tr} \pi(F)
$$

on $G$ and the Fourier transform (see Definition 2.1.3) of the orbital integral

$$
f \mapsto \int_{G / C_{G}\left(X^{*}\right)^{\circ}} f\left(\operatorname{Ad}^{*}(g) X^{*}\right) \mathrm{d} \dot{g}
$$

on $\mathfrak{g}^{*}$ are represented, at least on $G^{\text {rss }}$ and $\mathfrak{g}^{\text {rss }}$, respectively, by scalar functions $\Theta_{\pi}$ ([14, p. 99, Corollary] and [2, Theorem B.1.1]) and $\hat{\mu}_{X^{*}}^{G}$ [2, Theorem A.1.2], respectively. The function $\Theta_{\pi}$ is canonical, but normalising the function $\hat{\mu}_{X^{*}}^{G}$ requires making a choice of $G$ invariant measure on $G / C_{G}\left(X^{*}\right)^{\circ}$. We use the quotient of Waldspurger's canonical Haar measures [28, §I.4] on $G$ and its (reductive [6, Proposition 13.19]) subgroup $C_{G}\left(X^{*}\right)^{\circ}$. (These measures assign mass $|\bar{K}| \cdot|\operatorname{Lie}(\bar{K})|^{-1 / 2}$ to a parahoric subgroup $K$ with reductive quotient $\bar{K}$; see [10, $§ 5.1$, p. 835].) Put

$$
\Phi_{\pi}=\left|D_{G}^{\mathrm{red}}\right|^{1 / 2} \Theta_{\pi} \quad \text { and } \quad \hat{O}_{X^{*}}^{G}=\left|D_{G}^{\mathrm{red}}\left(X^{*}\right)\right|^{1 / 2}\left|D_{G}^{\mathrm{red}}\right|^{1 / 2} \hat{\mu}_{X^{*}}^{G},
$$


and, if $X^{*}$ is strongly regular semisimple, then also

$$
\widehat{S O}_{X^{*}}^{G}=\sum_{X^{\prime *} \in G \backslash \mathbf{G} \cdot X^{*}} \hat{O}_{X^{\prime *}}^{G},
$$

where $\mathbf{G} \cdot X^{*}:=\operatorname{Ad}^{*}\left(\mathbf{G}\left(k^{\text {un }}\right)\right) X^{*} \cap \mathfrak{g}^{*}$ is the $\mathbf{G}$-stable conjugacy class of $X^{*}$ (see [17, $\S 3$, p. 788] and [10, $\S 2.9$, p. 817]), as functions defined at least on $G^{\text {rss }}, \mathfrak{g}^{\text {rss }}$, and $\mathfrak{g}^{\text {rss }}$, respectively.

Remark 4.1.7. The functions $\Theta_{\pi}, \hat{O}_{X^{*}}^{G}$, and $\widehat{S O}_{X^{*}}^{G}$ may extend smoothly off the regular semisimple set; for example, they are globally defined if $G / Z(G)$ is compact. Since $G^{\text {rss }}$ is dense in $G$, any such extension is uniquely determined. Therefore, we do not distinguish notationally between the functions and their maximal smooth extensions.

For the remainder of this section, fix a compact, central, expanded cuspidal quintuple $\left(G^{\prime}, \pi^{\prime}, \phi, x, X^{*}\right)$. Let $r>0$ be the depth of the quintuple. Fix also an $r$-approximable element $\gamma \in G$, say with $r$-approximation $\gamma=\gamma_{<r} \gamma_{\geq r}$ [3, Definition 6.8]. Until 4.4, put $\mathbf{H}=C_{\mathbf{G}}^{(r)}(\gamma)$ [3, Definition 9.3]. (In $\S \varangle 4.4$ and 4.5, the connected group $C_{\mathbf{G}}^{(r)}(\gamma)$ is enlarged to the possibly disconnected group $C_{\mathbf{G}}\left(\gamma_{<r}\right)$, of which $C_{\mathbf{G}}^{(r)}(\gamma)$ is the identity component [3, Corollary 6.14].)

Let $\mathbf{T}$ be a maximal $H$-torus (so that $\gamma_{<r} \in T$ ), and write

- $R=R(\mathbf{G}, \mathbf{T})$,

- $\widetilde{R}=\widetilde{R}(\mathbf{G}, \mathbf{T})$,

- $R_{\mathbf{H}}=R(\mathbf{H}, \mathbf{T})$, and

- $\widetilde{R}_{\mathbf{H}}=\widetilde{R}(\mathbf{H}, \mathbf{T})$.

It is convenient to impose no additional hypotheses on $\mathbf{T}$ for Lemma 4.1.9, but we do add some hypotheses immediately afterwards.

Remark 4.1.8. By [3, Lemma 6.13], if $\gamma \in T$, then $\gamma_{<r} \in T$ as well. In this case, we have that $\operatorname{ord}\left(\alpha(\gamma)-\alpha\left(\gamma_{<r}\right)\right) \geq r$ (hence that $\operatorname{ord}_{\gamma} \alpha=\operatorname{ord}_{\gamma_{<r}} \alpha$ ) for $\alpha \in \widetilde{R} \backslash \widetilde{R}_{\mathbf{H}}$, and that $\alpha(\gamma)=\alpha\left(\gamma_{\geq r}\right)$ for $\alpha \in \widetilde{R}_{\mathbf{H}}$. In particular, $\widetilde{R}_{\mathbf{H}}=\left\{\alpha \in \widetilde{R} \mid \operatorname{ord}_{\gamma} \alpha \geq r\right\}$.

Lemma 4.1.9. If $\gamma \in T$, then $\left|D_{G}^{\mathrm{red}}(\gamma)\right|=\left|D_{G}^{\mathrm{red}}\left(\gamma_{<r}\right)\right| \cdot\left|D_{H}^{\mathrm{red}}\left(\gamma_{\geq r}\right)\right|$.

Proof. By Remark 2.2.9, we have that

$$
\begin{array}{r}
\left|D_{G}^{\mathrm{red}}(\gamma)\right|=\prod_{\substack{\alpha \in R \\
\alpha(\gamma) \neq 1}} q^{-\operatorname{ord}_{\gamma} \alpha}, \quad\left|D_{G}^{\mathrm{red}}\left(\gamma_{<r}\right)\right|=\prod_{\substack{\alpha \in R \\
\alpha\left(\gamma_{<r}\right) \neq 1}} q^{-\operatorname{ord}_{\gamma_{<r}} \alpha}, \\
\text { and }\left|D_{H}^{\mathrm{red}}\left(\gamma_{\geq r}\right)\right|=\prod_{\substack{\alpha \in R_{\mathrm{H}} \\
\alpha\left(\gamma_{\geq r}\right) \neq 1}} q^{-\operatorname{ord}_{\gamma_{\geq}} \alpha},
\end{array}
$$

so that the desired equality follows from Remark 4.1.8.

For the remainder of this section, suppose that $\mathbf{T}$ is $k^{\text {tame }}$-split and contained in $\mathbf{G}^{\prime}$, and that $x \in \mathcal{B}(\mathbf{T}, k)$. Then $\gamma_{<r} \in T \subseteq G^{\prime}$ [3, Remark 6.10(1)], so that it makes sense to define $C_{\mathbf{G}^{\prime}}^{(r)}\left(\gamma_{<r}\right)$. By abuse of notation, we write $C_{\mathbf{G}^{\prime}}^{(r)}(\gamma)$, or just $\mathbf{H}^{\prime}$, in place of $C_{\mathbf{G}^{\prime}}^{(r)}\left(\gamma_{<r}\right)$. Put $R^{\prime}=R\left(\mathbf{G}^{\prime}, \mathbf{T}\right)$ and $R_{\mathbf{H}^{\prime}}=R\left(\mathbf{H}^{\prime}, \mathbf{T}\right)=R_{\mathbf{H}} \cap R^{\prime}$.

Remark 4.1.10. By [3, Lemma 6.5], we have that $\mathbf{H}^{\prime}=\mathbf{H} \cap \mathbf{G}^{\prime}$. 
In order to minimise notation, it helps to have a notation for the Fourier transforms of orbital integrals occurring in character formulæ that does not require us to refer directly to the element $X^{*}$.

Definition 4.1.11. Write $\hat{O}_{\pi^{\prime}}^{H}$ and (if $X^{*}$ is strongly regular semisimple, that is, if $\mathbf{G}^{\prime}$ is a torus) $\widehat{S O}_{\pi^{\prime}}^{H}$ for the restrictions to $\mathfrak{h}^{\text {rss }} \cap \mathfrak{h}_{r}$ of $\hat{O}_{X^{*}}^{H}$ and $\widehat{S O}_{X^{*}}^{H}$, respectively.

Since $\left.\pi^{\prime}\right|_{G_{x, r}}$ is $\left.\phi\right|_{G_{x, r}}$-isotypic, we have by [4, Lemma B.4] that $\hat{O}_{\pi^{\prime}}^{H}$ and $\widehat{S O}_{\pi^{\prime}}^{H}$ depend only on $\pi^{\prime}$ (indeed, only on $\left.\phi\right|_{G_{x, r}}$ ), not on the choice of expanded quintuple.

4.2. Indices. The character formulæ of [4] involve groups $\llbracket \gamma ; x, r \rrbracket$ and $\llbracket \gamma_{<r} ; x, r+\rrbracket$. These are defined in [3, Definition 6.6], but, since we are interested only in the cardinalities of certain quotients involving these groups, we do not reproduce their full definitions here (though see the proof of Proposition 4.2.1 below). Since the definitions of these groups depend only on $\gamma_{<r}$, we ease notation by assuming throughout this section that $\gamma=\gamma_{<r}$.

Proposition 4.2.1.

$$
\begin{aligned}
{\left[\llbracket \gamma ; x, r \rrbracket: T_{0+} G_{x, r / 2}\right] \cdot[\llbracket \gamma ; x, r+\rrbracket} & \left.: T_{0+} G_{x,(r / 2)+}\right] \\
& =\left|\mathfrak{t}_{0: 0+}\right| \cdot\left|\mathfrak{h}_{x, 0: 0+}\right|^{-1} \cdot \prod_{\alpha \in R_{\mathbf{H}}} q^{r} \cdot\left|D_{G}^{\mathrm{red}}(\gamma)\right|^{-1} .
\end{aligned}
$$

Proof. In the notation of [3, Definition 5.14], write ${ }_{\mathbf{T}} G_{x, 0+\vee}\left(r-\operatorname{ord}_{\gamma}\right) / 2$ and $\mathbf{T}_{\mathbf{T}} \mathfrak{g}_{x, 0+\vee}\left(r-\operatorname{ord}_{\gamma}\right) / 2$ as abbreviations for ${ }_{\mathbf{T}} G_{x, f_{\mathbf{H}}}$ and ${ }_{\mathbf{T}} \mathfrak{g}_{x, f_{\mathbf{H}}}$, where

$$
f_{\mathbf{H}}(\alpha)= \begin{cases}0+, & \alpha \in \widetilde{R}_{\mathbf{H}} \\ \frac{1}{2}\left(r-\operatorname{ord}_{\gamma} \alpha\right), & \alpha \in \widetilde{R} \backslash \widetilde{R}_{\mathbf{H}} ;\end{cases}
$$

and, similarly, ${ }_{\mathbf{T}} G_{x,\left(0 \vee\left(r-\operatorname{ord}_{\gamma}\right) / 2\right)+}$ and ${ }_{\mathbf{T}} \mathfrak{g}_{x,\left(0 \vee\left(r-\operatorname{ord}_{\gamma}\right) / 2\right)+}$ instead of ${ }_{\mathbf{T}} G_{x, f+}$ and $\mathbf{T}_{\mathbf{T}} \mathfrak{g}_{x, f+}$, where $f+$ is defined by

$$
(f+)(\alpha)=f_{\mathbf{H}}(\alpha)+= \begin{cases}0+, & \alpha \in \widetilde{R}_{\mathbf{H}} \\ \frac{1}{2}\left(r-\operatorname{ord}_{\gamma} \alpha\right)+, & \alpha \in \widetilde{R} \backslash \widetilde{R}_{\mathbf{H}} .\end{cases}
$$

Note that $f_{\mathbf{H}}$ (hence also $f+$ ) is everywhere strictly positive. We are also interested in the merely non-negative function $f$ defined by

$$
f(\alpha)= \begin{cases}0, & \alpha \in \widetilde{R}_{\mathbf{H}} \\ \frac{1}{2}\left(r-\operatorname{ord}_{\gamma} \alpha\right), & \alpha \in \widetilde{R} \backslash \widetilde{R}_{\mathbf{H}},\end{cases}
$$

and, as above, we write $\mathfrak{T}_{x, 0 \vee}\left(r-\operatorname{ord}_{\gamma}\right) / 2$ instead of ${ }_{\mathbf{T}} \mathfrak{g}_{x, f}$. (The corresponding group is not of interest to us.)

By [3, Definition 6.6],

$$
\llbracket \gamma ; x, r \rrbracket={ }_{\mathbf{T}} G_{x, 0+\vee\left(r-\operatorname{ord}_{\gamma}\right) / 2} \quad \text { and } \quad \llbracket \gamma ; x, r+\rrbracket={ }_{\mathbf{T}} G_{\left.x, 0 \vee\left(r-\operatorname{ord}_{\gamma}\right) / 2\right)+} \cdot
$$

In the notation of [3, Definition 5.14], we have by [3, Proposition 5.40] that $T_{0+} G_{x, r / 2}=$ $(T, G)_{x,(0+, r / 2)}$ and $T_{0+} G_{x,(r / 2)+}=(T, G)_{x,(0+,(r / 2)+)}$; the corresponding Lie-algebra statements follow immediately from Definition 3.2.1. Thus, we are almost in a position to use Lemma 3.2.5 except for two obstacles. First, we are working on the group, not on the Lie algebra; and, second, the function $f_{\mathbf{H}}$ is not real valued.

The solution proceeds in two stages: we first pass to the Lie algebra, then 'manually' account for the difference between $f_{\mathbf{H}}$ and the real-valued function $f$. Although there need not be any reasonably behaved map from ${ }_{\mathbf{T}} G_{x,\left(0+\vee\left(r-\operatorname{ord}_{\gamma}\right) / 2\right)} / T_{0+} G_{x, r / 2}$ to 
$\mathbf{T} \mathfrak{g}_{x,\left(0+\vee\left(r-\operatorname{ord}_{\gamma}\right) / 2\right)} /\left(\mathfrak{t}_{0+}+\mathfrak{g}_{x, r / 2}\right)$, an argument as in [3, Corollary 5.25] shows that there are filtrations $\left(\mathcal{G}_{i}\right)_{i=0}^{n}$ and $\left(\operatorname{Lie}\left(\mathcal{G}_{i}\right)\right)_{i=0}^{n}$ of ${ }_{\mathbf{T}} G_{x, 0+\vee\left(r-\operatorname{ord}_{\gamma}\right) / 2}$ and ${ }_{\mathbf{T}} \mathfrak{g}_{x, 0+\vee}\left(r-\operatorname{ord}_{\gamma}\right) / 2$, respectively, such that

- $\mathcal{G}_{i} / \mathcal{G}_{i+1} \cong \operatorname{Lie}\left(\mathcal{G}_{i}\right) / \operatorname{Lie}\left(\mathcal{G}_{i+1}\right)$ for all $i \in\{0, \ldots, n-1\}$ (via a Moy-Prasad isomorphism; see [19, §3.7] and [1, §1.5, p. 12]),

- $\mathcal{G}_{0}={ }_{\mathbf{T}} G_{x, 0+\vee}\left(r-\operatorname{ord}_{\gamma}\right) / 2$ and $\operatorname{Lie}\left(\mathcal{G}_{0}\right)={ }_{\mathbf{T}} \mathfrak{g}_{x, 0+\vee}\left(r-\operatorname{ord}_{\gamma}\right) / 2$, and

- $\mathcal{G}_{n}=T_{0+} G_{x, r / 2}$ and $\operatorname{Lie}\left(\mathcal{G}_{n}\right)=\mathfrak{t}_{0+}+\mathfrak{g}_{x, r / 2}$.

Thus,

$$
\left[\llbracket \gamma ; x, r \rrbracket: T_{0+} G_{x, r / 2}\right]=\left[\mathbf{T} \mathfrak{g}_{x,\left(0+\vee\left(r-\operatorname{ord}_{\gamma}\right) / 2\right)}: \mathfrak{t}_{0+}+\mathfrak{g}_{x, r / 2}\right]
$$

and, similarly,

$$
\left[\llbracket \gamma ; x, r+\rrbracket: T_{0+} G_{x,(r / 2)+}\right]=\left[\mathbf{T g}_{x,\left(0 \vee\left(r-\operatorname{ord}_{\gamma}\right) / 2\right)+}: \mathfrak{t}_{0+}+\mathfrak{g}_{x,(r / 2)+}\right],
$$

so that we may re-write the left-hand side of the equality in the statement as

$$
\begin{aligned}
\underbrace{\left[\mathfrak{t}_{0}+\mathfrak{g}_{x, r / 2}: \mathfrak{t}_{0+}+\mathfrak{g}_{x, r / 2}\right] \cdot\left|\mathbf{T} \mathfrak{g}_{x,\left(0 \vee\left(r-\operatorname{ord}_{\gamma}\right) / 2\right):\left(0+\vee\left(r-\operatorname{ord}_{\gamma}\right) / 2\right)}\right|^{-1}}_{(*)} \times \\
\underbrace{\left[\mathbf{T} \mathfrak{g}_{x,\left(0 \vee\left(r-\operatorname{ord}_{\gamma}\right) / 2\right)}: \mathfrak{t}_{0}+\mathfrak{g}_{x, r / 2}\right] \cdot\left[\mathbf{T} \mathfrak{g}_{x,\left(0 \vee\left(r-\operatorname{ord}_{\gamma}\right) / 2\right)+}: \mathfrak{t}_{0+}+\mathfrak{g}_{x,(r / 2)+}\right]}_{(* *)} .
\end{aligned}
$$

Since $\frac{1}{2}\left(r-\operatorname{ord}_{\gamma} \alpha\right) \leq 0$ exactly when $\operatorname{ord}_{\gamma} \alpha \geq r$, i.e., exactly when $\alpha \in \widetilde{R}_{\mathbf{H}}$ (see Remark 4.1.8), we have

$$
(*)=\left|\mathfrak{t}_{0: 0+}\right| \cdot\left|\mathfrak{h}_{x, 0: 0+}\right|^{-1} .
$$

By Lemma 3.2.5 (and Remark 3.2.6) and [3, Corollary 6.14], we have

$$
\begin{aligned}
(* *) & =\prod_{\alpha \in R_{\mathbf{H}}} q^{2(r / 2-0)} \cdot \prod_{\alpha \in R \backslash R_{\mathbf{H}}} q^{2\left[r / 2-\left(r-\operatorname{ord}_{\gamma} \alpha\right) / 2\right]} \\
& =\prod_{\alpha \in R_{\mathbf{H}}} q^{r} \cdot \prod_{\alpha \in R \backslash R_{\mathbf{H}}} q^{\operatorname{ord}_{\gamma} \alpha} \\
& =\prod_{\alpha \in R_{\mathbf{H}}} q^{r} \cdot\left|D_{G}^{\mathrm{red}}(\gamma)\right|
\end{aligned}
$$

\section{Corollary 4.2.2.}

$$
\begin{aligned}
& {\left[\llbracket \gamma ; x, r \rrbracket: \llbracket \gamma ; x, r \rrbracket_{G^{\prime}} G_{x, r / 2}\right] \cdot\left[\llbracket \gamma ; x, r+\rrbracket: \llbracket \gamma ; x, r+\rrbracket_{G^{\prime}} G_{x,(r / 2)+}\right]} \\
& \quad=\left|\mathfrak{h}_{x, 0: 0+}\right|^{-1}\left|\mathfrak{h}_{x, 0: 0+}^{\prime}\right| \cdot\left|D_{H}^{\mathrm{red}}\left(X^{*}\right)\right| \cdot\left|D_{G}^{\mathrm{red}}(\gamma)\right|^{-1}\left|D_{G^{\prime}}^{\mathrm{red}}(\gamma)\right| .
\end{aligned}
$$

Proof. By [3, Proposition 5.40 and Lemma 5.29], we have that $\llbracket \gamma ; x, d \rrbracket_{G^{\prime}} \cap T_{0+} G_{x, d / 2}=$ $T_{0+} G_{x, d / 2}^{\prime}$ for $d \in\{r, r+\}$; so the left-hand side of the equality in the statement is

$$
\begin{aligned}
& \left(\left[\llbracket \gamma ; x, r \rrbracket: T_{0+} G_{x, r / 2}\right] \cdot\left[\llbracket \gamma ; x, r+\rrbracket: T_{0+} G_{x,(r / 2)+}\right]\right) \times \\
& \left(\left[\llbracket \gamma ; x, r \rrbracket_{G^{\prime}}: T_{0+} G_{x, r / 2}^{\prime}\right] \cdot\left[\llbracket \gamma ; x, r+\rrbracket_{G^{\prime}}: T_{0+} G_{x,(r / 2)+}^{\prime}\right]\right)^{-1} .
\end{aligned}
$$

By Proposition 4.2.1, this equals

$$
\left|\mathfrak{h}_{x, 0: 0+}\right|^{-1}\left|\mathfrak{h}_{x, 0: 0+}^{\prime}\right| \cdot \underbrace{\prod_{\alpha \in R_{\mathbf{H}} \backslash R_{\mathbf{H}^{\prime}}} q^{r}}_{(*)} \cdot\left|D_{G}^{\mathrm{red}}(\gamma)\right|^{-1}\left|D_{G^{\prime}}^{\mathrm{red}}(\gamma)\right| .
$$


By Remark 4.1.10, we have that $\mathbf{H}^{\prime}=\mathbf{H} \cap \mathbf{G}^{\prime}=C_{\mathbf{H}}\left(X^{*}\right)$. Since $R_{\mathbf{H}^{\prime}}=R_{\mathbf{H}} \cap R^{\prime}$, we have by [29, $\S 8$, GE1] that $\operatorname{ord}_{X^{*}} \alpha=-r$ for the roots $\alpha$ in the product $(*)$; so

$$
(*)=\prod_{\alpha \in R_{\mathbf{H}} \backslash R_{\mathbf{H}^{\prime}}} q^{-\operatorname{ord}_{X^{*} \alpha}}=\left|D_{H}^{\mathrm{red}}\left(X^{*}\right)\right| .
$$

4.3. Roots of unity. The character formulæ of [4] involve 4th roots of unity defined in terms of Galois actions on root systems. Definition 4.3.1 below breaks down these roots in a slightly different way from what is done in [4]; in Proposition 4.3.8, we show that the two approaches give the same result.

Except for the symbols whose definitions make explicit reference to the group $\mathbf{H}=$ $C_{\mathbf{G}}^{(r)}(\gamma)$, the definitions below do not depend on the approximability of $\gamma$.

Definition 4.3.1. Write

- $R_{x, r / 2}=\left\{\alpha \in R \mid r / 2 \in \operatorname{ord}_{x}(\alpha)\right\}$,

- $R_{x,\left(r-\operatorname{ord}_{\gamma}\right) / 2}=\left\{\alpha \in R \mid \alpha(\gamma) \neq 1\right.$ and $\left.\left(r-\operatorname{ord}_{\gamma} \alpha\right) / 2 \in \operatorname{ord}_{x}(\alpha)\right\}$, and

- $R\left(\pi^{\prime}, \gamma\right)=R_{x,\left(r-\operatorname{ord}_{\gamma}\right) / 2} \backslash R^{\prime}$.

As in [4, Proposition 5.2.13], write also

- $\mathfrak{G}=q^{-1 / 2} \sum_{t \in \mathfrak{f}} \Lambda\left(t^{2}\right)$;

- $t_{\alpha}=\frac{1}{2} e_{\alpha} \cdot \mathrm{N}_{k_{\alpha} / k_{ \pm \alpha}}\left(w_{\alpha}\right) \cdot \mathrm{d} \alpha^{\vee}\left(X^{*}\right) \cdot(\alpha(\gamma)-1)$, where $w_{\alpha}$ is an element of $k_{\alpha}$ of valuation $\frac{1}{2}\left(r-\operatorname{ord}_{\gamma} \alpha\right)$ such that $w_{\alpha}^{2} \in k_{ \pm \alpha}$, for $\alpha \in R\left(\pi^{\prime}, \gamma\right)$; and

- $\mathbf{G}_{ \pm \alpha}$ for the $k_{ \pm \alpha}$-group generated by the ( $\left.\pm \alpha\right)$-root subgroups of $\mathbf{G}$.

Now we introduce a number of new pieces of notation, each of which builds on the others. If $A$ is a finite, Abelian group of even order, then denote by $\operatorname{sgn}_{A}$ the unique non-trivial homomorphism from $A$ to $\{ \pm 1\}$. First put

$$
\begin{aligned}
\tilde{e}(G, T, \gamma) & =(-1)^{\left.\mid \Gamma \backslash R_{x,(r-\mathrm{ord} \gamma}\right) / 2 \mid}, \\
\varepsilon_{\text {symm, unram }}(G, T, \gamma) & =\prod_{\alpha \in \Gamma \backslash R_{\text {symm, unram }, x, r / 2}} \operatorname{sgn}_{\mathfrak{f}_{\alpha}^{1}}(\alpha(\gamma)), \\
\varepsilon_{x, r / 2}^{\text {symm }}(G, T, \gamma) & =\prod_{\alpha \in \pm \Gamma \backslash R_{x, r / 2}^{\text {symm }}} \operatorname{sgn}_{\mathfrak{f}_{\alpha}}(\alpha(\gamma)),
\end{aligned}
$$

and

$$
\varepsilon_{x, r / 2}^{\mathrm{ram}}(G, T, \gamma)=\varepsilon_{x, r / 2}^{\mathrm{symm}}(G, T, \gamma) \varepsilon_{\mathrm{symm}, \text { unram }}(G, T, \gamma)
$$

Next put

$$
\tilde{e}\left(G / G^{\prime}, \gamma\right)=\frac{\tilde{e}(G, T, \gamma)}{\tilde{e}\left(G^{\prime}, T, \gamma\right)}
$$

and similarly for $\varepsilon_{\text {symm, unram }}, \varepsilon_{x, r / 2}^{\mathrm{symm}}$, and $\varepsilon_{x, r / 2}^{\mathrm{ram}}$; and then

$$
e\left(G / G^{\prime}, \gamma\right)=\prod_{\substack{\alpha \in R \backslash R^{\prime} \\ \alpha(\gamma) \neq 1}}(-1)^{e_{\alpha}\left(r-\operatorname{ord}_{\gamma} \alpha\right)}
$$


and

$$
\begin{array}{r}
\varepsilon_{\text {symm, ram }}\left(G / G^{\prime}, \gamma\right)=\prod_{\alpha \in \Gamma \backslash R\left(\pi^{\prime}, \gamma\right)_{\text {symm, ram }}}(-1)^{\mathrm{rank}_{ \pm \alpha} \mathbf{G}_{ \pm \alpha}-1}(-\mathfrak{G})^{f_{\alpha}} \times \\
\operatorname{sgn}_{\mathfrak{f}_{\alpha}}\left(t_{\alpha}\right) \operatorname{sgn}_{k_{ \pm \alpha}}\left(\mathbf{G}_{ \pm \alpha}\right) .
\end{array}
$$

Finally, put

$$
\tilde{e}\left(\pi^{\prime}, \gamma\right)=\frac{\tilde{e}\left(G / G^{\prime}, \gamma\right)}{\tilde{e}\left(H / H^{\prime}, \gamma\right)},
$$

and similarly for $\varepsilon_{\text {symm, unram }}, \varepsilon^{\text {symm }}, \varepsilon^{\text {ram }}, \varepsilon_{\text {symm, ram }}$, and $e$.

The signs occurring in Definition 4.3.1 play an important rôle in the character computations, and their behaviour is surprisingly subtle (for example, it necessitates the modification of the original Yu construction described in Definition 5.4 below); so we take a while to discuss some of their important properties.

Remark 4.3.2. The riot of notation in Definition 4.3.1 serves a purpose; each term is handled differently. The function $\varepsilon^{\mathrm{ram}}\left(\pi^{\prime}\right): \gamma \mapsto \varepsilon^{\mathrm{ram}}\left(\pi^{\prime}, \gamma\right)$ is a character of $T$. We build it into the 'twisted' construction (Definition 5.4) of supercuspidals from toral pairs. The function $\tilde{e}\left(\pi^{\prime}\right)$ is the source of the sign changes predicted by Kottwitz [18]; see Proposition 4.3.7. The function $\varepsilon_{\text {symm, ram }}\left(\pi^{\prime}\right)$ is a bit more mysterious; but, by Lemma 4.3.6, it does not change under stable conjugacy, and so, for our purposes, this mystery may be swept under the rug.

Remark 4.3.3. If $\mathbf{T}$ is an elliptic $G$-torus and $\alpha \in R$, then $\sum_{\sigma \in \Gamma / \Gamma_{\alpha}} \alpha$ is a (rational) character of $\mathbf{T} / Z(\mathbf{G})$, hence is trivial; so

$$
\begin{aligned}
\operatorname{sgn}_{\mathfrak{f}_{\alpha}}(\alpha(\gamma))^{e_{\alpha}} & =\operatorname{sgn}_{\mathfrak{f}}\left(N_{\mathfrak{f}_{\alpha} / \mathfrak{f}}(\alpha(\gamma))\right)^{e_{\alpha}} \\
& =\operatorname{sgn}_{\mathfrak{f}}\left(N_{k_{\alpha} / k}(\alpha(\gamma))\right) \\
& =\operatorname{sgn}_{\mathfrak{f}}\left(\left(\sum_{\sigma \in \Gamma / \Gamma_{\alpha}} \alpha\right)(\gamma)\right)=1 \quad \text { for all } \gamma \in T .
\end{aligned}
$$

In particular, if $e_{\alpha}$ is odd, then $\operatorname{sgn}_{\mathfrak{f}_{\alpha}} \circ \alpha=1$; so, if $\mathbf{T}$ splits over an extension of odd ramification degree, then the character $\varepsilon_{x, r / 2}^{\text {symm }}(G, T): \gamma \mapsto \varepsilon_{x, r / 2}^{\text {symm }}(G, T, \gamma)$ of Definition 4.3.1 is trivial.

Remark 4.3.4. The quantities $\tilde{e}(G, T, \gamma)$, $\varepsilon_{\text {symm, unram }}(G, T, \gamma)$, and $\varepsilon_{\text {symm, ram }}\left(G / G^{\prime}, \gamma\right)$ all depend on $r$, although we suppress it from the notation for convenience.

By Corollary 3.1.8, we have that $\tilde{e}(G, T, \gamma)=(-1)^{\mid \Gamma \backslash R_{x,(r-\operatorname{ord} \gamma) / 2, \text { symm }}}$.

The restriction of a symmetric root $\alpha$ to the maximal $k$-split subtorus $\mathbf{T}_{\mathrm{s}}$ of $\mathbf{T}$ is both fixed (because $\mathbf{T}_{\mathrm{s}}$ is $k$-split) and negated (because $\alpha$ is symmetric) by some element of $\Gamma$; so $\alpha$ is trivial on $\mathbf{T}_{\mathrm{s}}$. That is, $R(\mathbf{G}, \mathbf{T})_{\text {symm }}=R(\mathbf{M}, \mathbf{T})_{\text {symm }}$, where $\mathbf{M}=C_{\mathbf{G}}\left(\mathbf{T}_{\mathrm{s}}\right)$. Since $\mathbf{T}$ is $k$-elliptic in $\mathbf{M}$, the image of $\mathcal{B}(\mathbf{T}, k)$ in $\mathcal{B}^{\text {red }}(\mathbf{M}, k)$ is a singleton; so it follows that $\tilde{e}(G, T, \gamma), \varepsilon_{\text {symm, unram }}(G, T, \gamma)$, and $\varepsilon_{\text {symm, ram }}\left(G / G^{\prime}, \gamma\right)$ do not depend on the choice of $x \in \mathcal{B}(\mathbf{T}, k)$.

By Remark 4.1.8, we have that $\tilde{e}\left(\pi^{\prime}, \gamma\right)$ depends only on $\gamma_{<r}$ (and $\pi^{\prime}$, and, possibly, $\left.\mathbf{T}\right)$, not on $\gamma$; and similarly for $\varepsilon_{\text {symm, unram }}, \varepsilon_{x, r / 2}^{\text {symm }}, e, \varepsilon_{\text {symm, ram }}$, and $\varepsilon_{x, r / 2}^{\text {ram }}$.

It seems likely that $\tilde{e}\left(G / G^{\prime}, \gamma\right)$, but not $\tilde{e}(G, T, \gamma)$, is independent of the choice of $k^{\text {tame }}$-split maximal torus $\mathbf{T}$ in $\mathbf{G}^{\prime}$ containing $\gamma$, and similarly for $\varepsilon_{\text {symm, unram }}, \varepsilon_{x, r / 2}^{\text {symm }}$, $\varepsilon_{\text {symm, ram }}$, and $\varepsilon_{x, r / 2}^{\mathrm{ram}}$. However, we currently do not have a proof of this. (It is clear 
that $e\left(G / G^{\prime}, \gamma\right)$ is independent of the choice of $\mathbf{T}$.) This uncertainty seems to have the potential to affect our character formulæ (Theorem 4.6.2 and Theorem 5.8). However, by [4, Propositions 3.8 and 5.2.13] (and [29, §11] and [4, Definition 5.2.4]), at least the product $\varepsilon_{\text {symm, ram }}\left(\pi^{\prime}, \gamma_{<r}\right) \varepsilon^{\mathrm{ram}}\left(\pi^{\prime}, \gamma_{<r}\right) \tilde{e}\left(\pi^{\prime}, \gamma_{<r}\right)$ that occurs in Theorem 4.6.2] is independent of $\mathbf{T}$; and, in Theorem 5.8 (and, indeed, throughout $₫ 5$ ), we are dealing with the case where $\mathbf{G}^{\prime}$ is a torus, so that there is only one possible choice of $\mathbf{T}$.

All the signs are invariant under rational conjugacy (Remark 4.3.5). Even better, $e$ and $\varepsilon_{\text {symm, ram }}$ are invariant under stable conjugacy (Lemma 4.3.6).

Remark 4.3.5. If $g \in G$, and we put

- $x^{\prime}=g \cdot x$,

- $\mathbf{T}^{\prime}=\operatorname{Int}(g) \mathbf{T}$, and

- $\gamma^{\prime}=\operatorname{Int}(g) \gamma$,

then it is clear that

$$
\begin{aligned}
\tilde{e}(G, T, \gamma) & =\tilde{e}\left(G, T^{\prime}, \gamma^{\prime}\right), \\
\varepsilon_{\text {symm, unram }}(G, T, \gamma) & =\varepsilon_{\text {symm, unram }}\left(G, T^{\prime}, \gamma^{\prime}\right),
\end{aligned}
$$

and

$$
\varepsilon_{x^{\prime}, r / 2}^{\mathrm{symm}}\left(G, T^{\prime}, \gamma^{\prime}\right)=\varepsilon_{x, r / 2}^{\mathrm{symm}}(G, T, \gamma)
$$

Lemma 4.3.6. If $g \in \mathbf{G}\left(k^{\mathrm{un}}\right)$ is such that $g^{-1} \operatorname{Fr} g \in \mathbf{T}\left(k^{\text {un }}\right)$, then $e\left(G / G^{\prime}, \gamma\right)=e\left(G / G^{\prime}, \operatorname{Int}(g) \gamma\right)$ and $\varepsilon_{\text {symm, ram }}\left(G / G^{\prime}, \gamma\right)=\varepsilon_{\text {symm, ram }}\left(G / G^{\prime}, \operatorname{Int}(g) \gamma\right)$.

Proof. Put $\mathbf{T}^{\prime}=\operatorname{Int}(g) \mathbf{T}$ and $\gamma^{\prime}=\operatorname{Int}(g) \gamma$. Note that the group $\mathbf{T}^{\prime}$ and the map $\operatorname{Int}(g)$ : $\mathbf{T} \rightarrow \mathbf{T}^{\prime}$ are defined over $k$; in particular, $\gamma^{\prime} \in T^{\prime}$. The equality for $e$ is clear, so we consider only the one for $\varepsilon_{\text {symm, ram }}$.

The element $g$ induces a natural, $\Gamma$-equivariant bijection $R(\mathbf{G}, \mathbf{T}) \rightarrow R\left(\mathbf{G}, \mathbf{T}^{\prime}\right)$, which we denote by $\alpha \mapsto \alpha^{\prime}$, such that $\alpha(\gamma)=\alpha^{\prime}\left(\gamma^{\prime}\right)$ for all $\alpha \in R(\mathbf{G}, \mathbf{T})$. Since it is $\Gamma$ equivariant, this identification preserves the notion of symmetry of a root, and of (un)ramifiedness of a symmetric root.

Choose $y \in \mathcal{B}\left(\mathbf{T}, k^{\text {un }}\right)$ such that $g \cdot y \in \mathcal{B}\left(\mathbf{T}^{\prime}, k^{\text {un }}\right)$ is $\Gamma$-fixed, i.e., lies in $\mathcal{B}\left(\mathbf{T}^{\prime}, k\right)$. We claim that, for any symmetric and ramified $\alpha \in R(\mathbf{G}, \mathbf{T})$, we have $\operatorname{ord}_{x} \alpha=\operatorname{ord}_{y} \alpha$. Note that $\alpha$ remains symmetric and ramified after an unramified base change. Further, by Remark 3.1.4 the sets $\operatorname{ord}_{x} \alpha$ and $\operatorname{ord}_{y} \alpha$ are not affected by such a change; so we may, and do, assume that $x, y \in \mathcal{B}(\mathbf{T}, k)=\mathcal{A}\left(\mathbf{T}_{k^{\text {sep }}}\right)^{\Gamma}$. Since $\mathcal{A}\left(\mathbf{T}_{k^{\text {sep }}}\right)$ is a torsor under $\mathbf{X}_{*}(\mathbf{T}) \otimes_{\mathbb{Z}} \mathbb{R}$, where $\mathbf{X}_{*}(\mathbf{T})$ is the cocharacter lattice of $\mathbf{T}_{k^{\text {sep }}}$, there is a well defined, $\Gamma$-fixed element $\lambda \in \mathbf{X}_{*}(\mathbf{T}) \otimes_{\mathbb{Z}} \mathbb{R}$ such that $x+\lambda=y$. Then

$$
2\langle\alpha, \lambda\rangle=\left\langle\left(1-\sigma_{\alpha}\right) \alpha, \lambda\right\rangle=\left\langle\alpha,\left(1-\sigma_{\alpha}^{-1}\right) \lambda\right\rangle=\langle\alpha, 0\rangle=0
$$

as desired. It is clear that also $\operatorname{ord}_{y} \alpha=\operatorname{ord}_{g \cdot y} \alpha^{\prime}$ (for any root $\alpha$, whether or not symmetric and ramified). Thus, the identification of $R(\mathbf{G}, \mathbf{T})$ with $R\left(\mathbf{G}, \mathbf{T}^{\prime}\right)$ establishes a

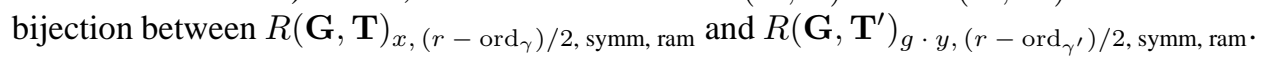
The result follows.

Proposition 4.3.7 below is the crucial ingredient in our proof of the stability of certain character sums (Theorem 5.10).

Proposition 4.3.7. If $\mathbf{G}^{\prime}$ contains a maximally $k^{\mathrm{un}}$-split torus in $\mathbf{G}$, then

$$
\tilde{e}\left(\pi^{\prime}, \gamma\right)=(-1)^{\mathrm{rank}_{k} \mathbf{G}-\operatorname{rank}_{k} \mathbf{G}^{\prime}} \cdot(-1)^{\operatorname{rank}_{k} \mathbf{H}-\operatorname{rank}_{k} \mathbf{H}^{\prime}} \cdot e\left(\pi^{\prime}, \gamma\right) .
$$


Proof. We may, and do, assume that $\gamma=\gamma_{<r}$.

If $\alpha \notin R^{\prime} \cup R_{\mathbf{H}}$, then we have that $r-\operatorname{ord}_{\gamma} \alpha=\operatorname{ord} \mathrm{d} \alpha^{\vee}\left(X^{*}\right)-\operatorname{ord}(\alpha(\gamma)-1) \in \mathbb{Z}_{\alpha}$. By Corollary 3.1.9, therefore, $\frac{1}{2}\left(r-\operatorname{ord}_{\gamma} \alpha\right) \in \operatorname{ord}_{x} \alpha$ if and only if $0 \in \operatorname{ord}_{x} \alpha$ and $r-\operatorname{ord}_{\gamma} \alpha \in 2 \mathbb{Z}_{\alpha}$, or $0 \notin \operatorname{ord}_{x} \alpha$ and $r-\operatorname{ord}_{\gamma} \alpha \notin 2 \mathbb{Z}_{\alpha}$. Thus,

$$
\tilde{e}\left(\pi^{\prime}, \gamma\right)=(-1)^{\left|\Gamma \backslash\left(R_{x} \backslash\left(R^{\prime} \cup R_{\mathbf{H}}\right)\right)\right|} \times \prod_{\alpha \in \Gamma \backslash\left(R \backslash\left(R^{\prime} \cup R_{\mathbf{H}}\right)\right)}(-1)^{e_{\alpha}\left(r-\operatorname{ord}_{\gamma} \alpha\right),}
$$

where $R_{x}=\left\{\alpha \in R \mid 0 \in \operatorname{ord}_{x} \alpha\right\}$. The result now follows from [23, Proposition 5.17].

Finally, we relate our roots of unity from Definition 4.3.1 to those occurring in [4]. Proposition 4.3 .8 and its proof may be challenging to read, because it is comparing two different systems of notation - the ad hoc system of [4], and the more nearly uniform system of this paper-that assign conflicting meanings to some symbols. We indicate explicitly when we are using the notation of [4].

Proposition 4.3.8. The quantity $\varepsilon(\phi, \gamma) \mathfrak{G}(\phi, \gamma)$ (in the notation of [4], Propositions 3.8 and 5.2.13]) equals $\varepsilon_{\mathrm{symm}, \mathrm{ram}}\left(\pi^{\prime}, \gamma\right) \varepsilon^{\mathrm{ram}}\left(\pi^{\prime}, \gamma\right) \tilde{e}\left(\pi^{\prime}, \gamma\right)$ (in the notation of Definition 4.3.1).

Proof. We may, and do, assume that $\gamma=\gamma_{<r}$.

Preserve the notation of Definition 4.3.1 There are defined in [4, Notations 3.7 and 5.2.11] disjoint sets $\Xi(\phi, \gamma)$ and $\Upsilon(\phi, \gamma)$ such that $\Xi(\phi, \gamma) \cup \Upsilon(\phi, \gamma)=R\left(\pi^{\prime}, \gamma\right)$. Those roots such that $\operatorname{ord}_{\gamma} \alpha=0$ lie in $\Xi(\phi, \gamma)$, and those such that $0<\operatorname{ord}_{\gamma} \alpha<r$ in $\Upsilon(\phi, \gamma)$. Note in particular that $\alpha(\gamma) \in 1+P_{\alpha}$ whenever $\alpha \in \Upsilon(\phi, \gamma)$. We abbreviate $\Xi(\phi, \gamma)$ to $\Xi$, and $\Upsilon(\phi, \gamma)$ to $\Upsilon$.

Now note that

$$
\begin{aligned}
& \varepsilon_{\text {symm, unram }}\left(\pi^{\prime}, \gamma\right)=\prod_{\alpha \in \Gamma \backslash\left(R_{x}, r / 2, \text { symm, unram } \backslash R^{\prime}\right)} \operatorname{sgn}_{\mathfrak{f}_{\alpha}^{1}}(\alpha(\gamma)) \\
& =\prod_{\alpha \in \Gamma \backslash \Xi_{\text {symm, urram }}} \operatorname{sgn}_{\mathfrak{f}_{\alpha}^{1}}(\alpha(\gamma)) \cdot \prod_{\begin{array}{c}
\alpha \in \Gamma \backslash\left(R_{x, r / 2, \text { symm, unram }} \backslash R^{\prime}\right) \\
\text { ord } \gamma \alpha>0
\end{array}}^{\operatorname{sgn}_{\mathfrak{f}_{\alpha}^{1}}(\alpha(\gamma))} \\
& =\prod_{\alpha \in \Gamma \backslash \Xi_{\text {symm, unram }}} \operatorname{sgn}_{\mathfrak{f}_{\alpha}^{1}}(\alpha(\gamma)) \cdot \prod_{\alpha \in \Gamma \backslash \Upsilon_{\text {symm, unram }}} \underbrace{\operatorname{sgn}_{\mathfrak{f}_{\alpha}^{1}}(\alpha(\gamma))}_{(*)} \\
& =\prod_{\alpha \in \Gamma \backslash R\left(\pi^{\prime}, \gamma\right)_{\text {symm, unram }}} \operatorname{sgn}_{\mathfrak{f}_{\alpha}^{1}}(\alpha(\gamma)),
\end{aligned}
$$

since each multiplicand $(*)$ equals 1 . Similarly,

$$
\varepsilon^{\mathrm{symm}}\left(\pi^{\prime}, \gamma\right)=\prod_{\alpha \in \Gamma \backslash R\left(\pi^{\prime}, \gamma\right)^{\mathrm{symm}}} \operatorname{sgn}_{\mathfrak{f}_{\alpha}}(\alpha(\gamma)) .
$$

We actually work in the proof with these products, rather than the ones appearing in Definition 4.3 .1

The proof of [4, Proposition 3.8] uses [11, Theorem 4.9.1]. As mentioned there, one could instead use [11, Corollary 4.8.1]; we do so here. Recall the decomposition

$$
\mathcal{V}=\mathcal{V}^{(0+)} \oplus \bigoplus_{\alpha \in \Gamma \backslash \Xi_{\mathrm{symm}}} V_{\alpha} \oplus \bigoplus_{\alpha \in \pm \Gamma \backslash \Xi^{\mathrm{symm}}} V_{ \pm \alpha}
$$


[4] (3.1)]. Then, in the notation of [4, Proposition 3.8] (and its proof), the quantity denoted there by $\varepsilon(\phi, \gamma)$ is the angular component of the complex number

$$
\theta_{W_{\zeta}^{\mathcal{\nu}^{(0+)}}}(\gamma) \cdot \prod_{\alpha \in \Gamma \backslash \Xi_{\mathrm{symm}}} \theta_{W_{\zeta}^{V_{\alpha}}}(\gamma) \cdot \prod_{\alpha \in \pm \Gamma \backslash \Xi^{\mathrm{symm}}} \theta_{W_{\zeta}^{V_{ \pm \alpha}}}(\gamma)
$$

[4, proof of Proposition 3.8, p. 1159, $(\dagger)$ ]. By [4, (3.3) and (3.4)], $\theta_{W_{\zeta}^{\nu^{(0+)}}}(\gamma)$ is a positive number, and

$$
\prod_{\alpha \in \pm \Gamma \backslash \Xi^{\mathrm{symm}}} \theta_{W_{\zeta}^{V_{ \pm \alpha}}}(\gamma)=\prod_{\alpha \in \pm \Gamma \backslash \Xi^{\mathrm{symm}}} \operatorname{sgn}_{\mathfrak{f}_{\alpha}}(\alpha(\gamma))=\varepsilon^{\mathrm{symm}}\left(\pi^{\prime}, \gamma\right)
$$

where the notation on the right is that of Definition 4.3.1 (and we have used that $\alpha(\gamma)$ is in $1+P_{\alpha}$ for $\left.\alpha \in \Upsilon\right)$. Thus, to understand the quantity $\varepsilon(\phi, \gamma)$ of [4], it remains only to compute the angular component of $\theta_{W_{\zeta}^{V_{\alpha}}}(\gamma)$ for $\alpha \in \Xi_{\text {symm }}$.

If $\alpha \in \Xi_{\mathrm{symm}, \text { ram }}$, then, as in [4] proof of Proposition 3.8, p. 1159], we obtain a nondegenerate pairing $t_{1} \otimes t_{2} \mapsto e_{\alpha} \operatorname{tr}_{\mathfrak{f}_{\alpha} / \mathbb{F}_{p}} c_{\alpha} \cdot t_{1} \sigma_{\alpha}\left(t_{2}\right)$ on $\mathfrak{f}_{\alpha}$, where $c_{\alpha} \in \mathfrak{f}_{\alpha}$ satisfies $\sigma_{\alpha} c_{\alpha}=-c_{\alpha}$. Since $\sigma_{\alpha} \in \Gamma^{\mathrm{un}}$ acts trivially on $\mathfrak{f}_{\alpha}$, and $p \neq 2$, we have that $c_{\alpha}=0$. Therefore, the non-degenerate pairing above is identically 0 , so its domain $V_{\alpha}$ is 0 . By definition [4, Notation 3.7], we have that $V_{\alpha}=\mathfrak{g}_{\alpha}\left(k_{\alpha}\right)_{x, r / 2:(r / 2)+}$; so, by Definition 3.1.3. this means that $r / 2=\frac{1}{2}\left(r-\operatorname{ord}_{\gamma} \alpha\right)$ does not lie in $\operatorname{ord}_{x} \alpha$, which is a contradiction.

Thus $\Xi_{\text {symm }}=\Xi_{\text {symm, unram. }}$. Choose $\alpha \in \Xi_{\text {symm, unram }}$, so that $f_{\alpha}=2 f_{ \pm \alpha}$. There is an $\mathfrak{f}$-linear isomorphism

$$
V_{\alpha} \cong \mathfrak{f}_{\alpha}
$$

that identifies the symplectomorphism $\gamma_{\alpha}$ of $V_{\alpha}$ induced by $\gamma$ with multiplication by $\alpha(\gamma)$; and there is an $\mathfrak{f}_{\alpha}$-split $\mathfrak{f}$-torus $\mathrm{S}_{\alpha}$ in $\mathrm{Sp}_{V_{\alpha}}$ such that an element $\bar{s} \in \mathrm{Sp}_{V_{\alpha}}(\mathfrak{f})$ lies in $\mathrm{S}_{\alpha}(\mathfrak{f})$ if and only if it is identified via (t†† with multiplication by an element of $\mathfrak{f}_{\alpha}^{1}$. In particular, $\gamma_{\alpha} \in \mathrm{S}_{\alpha}(\mathfrak{f})$.

In order to apply [11, Corollary 4.8.1], we need to understand the $\pm \Gamma$-orbits of weights of the action of $\mathrm{S}_{\alpha}$ on $V_{\alpha}$. Since $\sigma_{\alpha}$ acts on the character lattice $\mathbf{X}^{*}\left(\mathrm{~S}_{\alpha}\right)$ of $\left(\mathrm{S}_{\alpha}\right)_{\text {fsep }}$ by negation, every such weight is symmetric. Now, if $\Omega$ is any orbit of weights, then, in the notation of [11, Definition 4.6], $i(\Omega)=\frac{1}{2}|\Omega|$ is the least positive integer $i$ such that $\operatorname{Fr}^{i}$ acts by negation on $\Omega$. Now $\operatorname{Fr}^{f_{ \pm \alpha}} \in \sigma_{\alpha} \Gamma^{\mathrm{un}}$, so that $\operatorname{Fr}^{f_{ \pm \alpha}}$ and $\sigma_{\alpha}$ act the same on $\mathbf{X}^{*}\left(\mathrm{~S}_{\alpha}\right)$. It follows that $f_{ \pm \alpha}$ is an odd multiple of $i(\Omega)$, so that $f_{\alpha}=2 f_{ \pm \alpha}$ is an odd multiple of $|\Omega|=2 i(\Omega)$ (in particular, $f_{\alpha}$ and $|\Omega|$ have the same parity) and $q_{ \pm \alpha}=q^{f_{ \pm \alpha}}$ is an odd power of $q_{\Omega}:=q^{i(\Omega)}$. This means that the character $\chi_{\Omega}^{\mathrm{S}_{\alpha}}$ of [11, Lemma 4.6(2)(e)] is

$$
\bar{s} \mapsto \epsilon(\bar{s})^{\left(1+q_{\Omega}\right) / 2}=\epsilon(\bar{s})^{\left(1+q_{ \pm \alpha}\right) / 2}=\operatorname{sgn}_{\mathfrak{f}_{\alpha}^{1}}(\epsilon(\bar{s})),
$$

where $\epsilon$ is any element of $\Omega$. We have used that $\epsilon(\bar{s})^{\left(1+q_{\Omega}\right) / 2}$ is in $\{ \pm 1\}$ for all $\bar{s} \in \mathrm{S}_{\alpha}(\mathfrak{f})$, and that $q_{ \pm \alpha}+1=\left|\mathfrak{f}_{\alpha}^{1}\right|$. Finally, since there are $\operatorname{dim}_{\mathfrak{f}} V_{\alpha}=f_{\alpha}$ weights of $\mathrm{S}_{\alpha}$ on $V_{\alpha}$, and the size of each orbit has the same parity as $f_{\alpha}$, the number $l\left(V_{\alpha}, \mathrm{S}_{\alpha} ; \gamma_{\alpha}\right)$ of orbits of weights is odd. Since (††) identifies $\gamma_{\alpha}$ with multiplication by the projection to $\mathfrak{f}_{\alpha}$ of $\alpha(\gamma) \in \mathcal{O}_{\alpha}$, we have that $N\left(V_{\alpha} ; \gamma_{\alpha}\right):=\left.\frac{1}{2} \operatorname{dim} \operatorname{ker}\left(\gamma_{\alpha}-1\right)\right|_{V_{\alpha}}=0$ and, for any weight $\epsilon$, 
that $\gamma_{\alpha}^{\epsilon}$ is a Galois conjugate of $\alpha(\gamma)$. Thus, [11, Corollary 4.8.1] gives

$$
\begin{aligned}
\theta_{W_{\zeta}^{V_{\alpha}}}(\gamma) & =(-1)^{l\left(V_{\alpha}, \mathrm{S}_{\alpha} ; \gamma_{\alpha}\right)} q^{N\left(V_{\alpha} ; \gamma_{\alpha}\right)} \prod_{\Omega} \chi_{\Omega}^{\mathrm{S}_{\alpha}}\left(\gamma_{\alpha}\right) \\
& =\left(-\operatorname{sgn}_{\mathfrak{f}_{\alpha}^{1}}(\alpha(\gamma))\right)^{l\left(V_{\alpha}, \mathrm{S}_{\alpha} ; \gamma_{\alpha}\right)} \\
& =-\operatorname{sgn}_{\mathfrak{f}_{\alpha}^{1}}(\alpha(\gamma)) .
\end{aligned}
$$

That is, by $\boxplus$ and $(\dagger)$,

$$
\begin{aligned}
\varepsilon(\phi, \gamma) & =(-1)^{\left|\Gamma \backslash \Xi_{\mathrm{symm}}\right|} \cdot \varepsilon^{\mathrm{symm}}\left(\pi^{\prime}, \gamma\right) \cdot \prod_{\alpha \in \Gamma \backslash \Xi_{\mathrm{symm}, \text { unram }}} \operatorname{sgn}_{\mathfrak{f}_{\alpha}^{1}}(\alpha(\gamma)) \\
& =(-1)^{\left|\Gamma \backslash \Xi_{\mathrm{symm}}\right|} \cdot \varepsilon^{\mathrm{symm}}\left(\pi^{\prime}, \gamma\right) \cdot \varepsilon_{\mathrm{symm}, \text { unram }}\left(\pi^{\prime}, \gamma\right) \\
& =(-1)^{\left|\Gamma \backslash \Xi_{\mathrm{symm}}\right|} \cdot \varepsilon^{\mathrm{ram}}\left(\pi^{\prime}, \gamma\right),
\end{aligned}
$$

where the notation on the left is that of [4, Proposition 3.8], and that on the right is that of Definition 4.3.1 (and we have used again that $\alpha(\gamma) \in 1+P_{\alpha}$ for $\alpha \in \Upsilon$ ).

Now, since $R\left(\pi^{\prime}, \gamma\right)_{\text {symm, ram }}=\Upsilon_{\text {symm, ram }}$, [4, Proposition 5.2.13] says precisely that

$$
\mathfrak{G}(\phi, \gamma)=(-1)^{\left|\Gamma \backslash \Upsilon_{\text {symm }}\right|} \varepsilon_{\text {symm, ram }}\left(\pi^{\prime}, \gamma\right)
$$

where the notation on the left is that of [4, Proposition 5.2.13], and that on the right is that of Definition 4.3.1

The desired equality now follows from $\left(\ddagger_{0}\right)$ and $\left(\Phi_{>0}\right)$.

4.4. An indexing set. In this section and 4.5 below, we change notation, putting $\mathbf{H}=$ $C_{\mathbf{G}}\left(\gamma_{<r}\right)$ (not $\left.\mathbf{H}=C_{\mathbf{G}}^{(r)}(\gamma)=C_{\mathbf{G}}\left(\gamma_{<r}\right)^{\circ}\right)$ and $\mathbf{H}^{\prime}=C_{\mathbf{G}^{\prime}}\left(\gamma_{<r}\right)$. The reasoning of [3. Proposition 8.4] guarantees only that $\mathbf{H}^{\circ}$, not $\mathbf{H}$ itself, is independent of the choice of approximation $\gamma=\gamma_{<r} \gamma_{\geq r}$; but we soon reduce all relevant statements about $\mathbf{H}$ to statements about $\mathbf{H}^{\circ}$, so it doesn't matter.

The following set occurs implicitly in [4, Theorem 6.4], where it is the indexing set for a sum used in the computation of the character of $Y_{G^{\prime}}^{G} \pi^{\prime}$.

Definition 4.4.1. Put $\mathcal{C}\left(\pi^{\prime}, \gamma\right):=\left\{g \in G \mid \operatorname{Int}(g) \gamma_{<r} \in G^{\prime}\right.$ and $\left.\operatorname{Int}(g) \gamma_{\geq r} \in G_{x, r}\right\}$.

Write $\mathcal{C}$ for $\mathcal{C}\left(\pi^{\prime}, \gamma\right)$. Lemma 4.4.2 below is a technical result on the indexing set, proven in [3]; we are mostly interested in its consequences, Corollaries 4.4.3 and 4.4.4

Lemma 4.4.2 ([3, Lemma 9.10]). $\mathcal{C} \cap G_{x, 0+}=G_{x, 0+}^{\prime} H_{x, 0+}$.

Corollary 4.4.3. The natural projection

$$
\operatorname{stab}_{G^{\prime}}(\bar{x}) \backslash \mathcal{C} / H \rightarrow \operatorname{stab}_{G^{\prime}}(\bar{x}) G_{x, 0+} \backslash G_{x, 0+} \mathcal{C} / H
$$

is a bijection.

Proof. Let us call the map $i_{\gamma}$. It is clear that it is a surjection.

Fix $g \in \mathcal{C}$. Replacing $\gamma$ by $\operatorname{Int}(g) \gamma$ (hence $\gamma_{<r}$ by $\operatorname{Int}(g) \gamma_{<r}$ and $\gamma_{\geq r}$ by $\operatorname{Int}(g) \gamma_{\geq r}$ ) replaces $\mathbf{H}$ by $\operatorname{Int}(g) \mathbf{H}, \mathcal{C}$ by $\mathcal{C} g^{-1}$, and $i_{\gamma}$ by $c g^{-1} \mapsto i_{\gamma}(c) g^{-1}$; so it suffices to compute the fibre over $g=1$. If $c \in \mathcal{C}$ is such that $\operatorname{stab}_{G^{\prime}}(\bar{x}) G_{x, 0+} c H=i_{\gamma}(c)=$ $i_{\gamma}(1)=\operatorname{stab}_{G^{\prime}}(\bar{x}) G_{x, 0+} H$, then, since $G_{x, 0+}$ is normalised by $\operatorname{stab}_{G^{\prime}}(\bar{x})$, there exists $k \in G_{x, 0+} \in \operatorname{stab}_{G^{\prime}}(\bar{x}) c H$. Such an element $k$ lies in $\mathcal{C}\left(\pi^{\prime}, \gamma_{<r}\right)$ (not necessarily $\left.\mathcal{C}\left(\pi^{\prime}, \gamma\right)\right)$. By Lemma 4.4.2 it follows that $k \in G_{x, 0+}^{\prime} H_{x, 0+} \subseteq \operatorname{stab}_{G^{\prime}}(\bar{x}) H$, so that $\operatorname{stab}_{G^{\prime}}(\bar{x}) k H=\operatorname{stab}_{G^{\prime}}(\bar{x}) c H$ is the trivial double coset. 
Corollary 4.4.4. If $\gamma_{<r} \in G^{\prime}$, then

$$
\begin{aligned}
& \operatorname{stab}_{G^{\prime}}(\bar{x}) G_{x, 0+} \cap H=\operatorname{stab}_{H^{\prime}}(\bar{x}) H_{x, 0+} \\
& \qquad \text { and } \operatorname{stab}_{G^{\prime}}(\bar{x}) G_{x, 0+} \cap H^{\circ}=\operatorname{stab}_{H^{\prime} \circ}(\bar{x}) H_{x, 0+} .
\end{aligned}
$$

Proof. Suppose that $h \in \operatorname{stab}_{G^{\prime}}(\bar{x}) G_{x, 0+} \cap H$, and write $h=g^{\prime} k$, where $g^{\prime} \in \operatorname{stab}_{G^{\prime}}(\bar{x})$ and $k \in G_{x, 0+}$. As in the proof of Corollary 4.4.3, we have that $k \in \operatorname{stab}_{G^{\prime}}(\bar{x}) H \subseteq$ $\mathcal{C}\left(\pi^{\prime}, \gamma_{<r}\right) \cap$, hence by Lemma 4.4.2 that $k \in G_{x, 0+}^{\prime} H_{x, 0+}$. It follows that

$$
h=g^{\prime} k \in H \cap \operatorname{stab}_{G^{\prime}}(\bar{x}) H_{x, 0+}=\left(H \cap \operatorname{stab}_{G^{\prime}}(\bar{x})\right) H_{x, 0+}=\operatorname{stab}_{H^{\prime}}(\bar{x}) H_{x, 0+},
$$

whence the first containment.

For the second containment, observe that, since $H_{x, 0+} \subseteq H^{\circ}$ and $H^{\prime} \cap H^{\circ}=G^{\prime} \cap H^{\circ}=$ $H^{\prime \circ}$ (see Remark 4.1.10), we have

$$
\begin{aligned}
H^{\circ} \cap \operatorname{stab}_{G^{\prime}}(\bar{x}) G_{x, 0+}=H^{\circ} & \cap \operatorname{stab}_{H^{\prime}}(\bar{x}) H_{x, 0+} \\
& =\left(H^{\circ} \cap \operatorname{stab}_{H^{\prime}}(\bar{x})\right) H_{x, 0+}=\operatorname{stab}_{H^{\prime}}(\bar{x}) H_{x, 0+} .
\end{aligned}
$$

4.5. Orbital integrals. Preserve the notation of 4.4 In particular, $\mathbf{H}$ and $\mathbf{H}^{\prime}$ are the possibly disconnected groups $C_{\mathbf{G}}\left(\gamma_{<r}\right)$ and $C_{\mathbf{G}^{\prime}}\left(\gamma_{<r}\right)$, respectively. Proposition 4.5.1 relates orbital integrals on $H$ and $H^{\circ}$; but note that, as in Proposition 4.3.8, in addition to changing the domain of integration, we are also changing the normalisation of the measure with respect to which it is computed.

Proposition 4.5.1. We have that

$$
\hat{\mu}_{X^{*}}^{\operatorname{stab}_{H}(\bar{x})}=\left[\operatorname{stab}_{H^{\prime}}(\bar{x}): \operatorname{stab}_{\left.H^{\prime \circ}(\bar{x})\right]^{-1}\left|\left(\mathfrak{h}^{\prime}, \mathfrak{h}\right)_{x,(0,0):(0,0+)}\right|^{1 / 2} \times} \sum_{h \in \operatorname{stab}_{H}(\bar{x}) / \operatorname{stab}_{H^{\circ}}(\bar{x})} \hat{\mu}_{\mathrm{Ad}^{*}(h) X^{*}}^{\operatorname{stab}_{H}(\bar{x})}\right.
$$

and

$$
\begin{gathered}
\hat{\mu}_{X^{*}}^{H}=\left[H^{\prime}: H^{\prime \circ}\right]^{-1}\left|\left(\mathfrak{h}^{\prime}, \mathfrak{h}\right)_{x,(0,0):(0,0+)}\right|^{1 / 2} \times \\
\sum_{h \in H / H^{\circ}} \hat{\mu}_{\mathrm{Ad}^{*}(h) X^{*},}^{H^{\circ}}
\end{gathered}
$$

as functions on $\mathfrak{h}^{\text {rss }}$, where the orbital integrals on the left are normalised as in [4, Corollary 6.6], and those on the right as in Definition 4.1.6

See [4, (6.4)] for the definition of $\hat{\mu}_{X^{*}}^{\mathrm{stab}_{H}(\bar{x})}$. We do not reproduce it here, since we are really interested only in $\hat{\mu}_{X^{*}}^{H}$.

Proof. Since the arguments are essentially identical, we discuss only the integral over $H$.

As observed after the proof of [4, Theorem 6.4], we may use [4, Lemma B.2] to rewrite the Fourier transform of an orbital integral with respect to $H$, which is the group of $k$-rational points of a possibly disconnected group, as a sum of Fourier transforms of orbital integrals with respect to $H^{\circ}$, which is the group of $k$-rational points of a connected group.

Since $C_{H}\left(X^{*}\right)=H^{\prime}$, we have by [4] Lemma B.2] that

$$
\hat{\mu}_{X^{*}}^{H}=\left[H^{\prime}: H^{\prime \circ}\right]^{-1} \sum_{h \in H / H^{\circ}} \hat{\mu}_{\mathrm{Ad}^{*}(h)^{-1} X^{*}}^{H^{\circ}}
$$


Note, however, that the right-hand side is still normalised with respect to the measure on $H^{\circ} / H^{\prime \circ}$ inherited from its embedding in $H / H^{\prime}$. By Corollary 4.4.4] (and [4, Corollary 6.6 and $\S 2]$ ), this measure assigns mass 1 to

$$
\left(H \cap \operatorname{stab}_{G^{\prime}}(\bar{x}) G_{x, 0+}\right) H^{\prime} / H^{\prime}=H_{x, 0+} H^{\prime} / H^{\prime}
$$

-i.e., to the image in $H / H^{\prime}$ of $H_{x, 0+} \subseteq H^{\circ}$, which lies in (the image in $H / H^{\prime}$ of) $H^{\circ} / H^{\prime \circ}$.

On the other hand, since $H^{\prime \circ} \cap H_{x, 0+}=H_{x, 0+}^{\prime}$ by [3, Lemma 6.5 and Corollary 4.8], we have that the quotient of the canonical measures on $H^{\circ}$ and $H^{\prime \circ}$ (see Definition 4.1.6) assigns to the image in $H^{\circ} / H^{\prime \circ}$ of $H_{x, 0+}$ the quotient of the canonical measure

$$
\left[H_{x, 0}: H_{x, 0+}\right]^{-1} \cdot\left(\left|H_{x, 0: 0+}\right| \cdot\left|\mathfrak{h}_{x, 0: 0+}\right|^{-1 / 2}\right)=\left|\mathfrak{h}_{x, 0: 0+}\right|^{-1 / 2}
$$

of $H_{x, 0+}$ by the canonical measure $\left|\mathfrak{h}_{x, 0: 0+}^{\prime}\right|^{-1 / 2}$ of $H_{x, 0+}^{\prime}$. The result follows.

4.6. An inductive formula for normalised characters. As preparation for our character computation Theorem 4.6.2, which is the main result of this section, we present the complicated-looking Lemma 4.6.1, which just assembles all of our previous results on the comparison between the notations of [4] and of this paper. As with Proposition 4.3.8, it is important to keep in mind that there are two different systems of notation in play.

Recall that we have required that our expanded cuspidal quintuple $\left(G^{\prime}, \pi^{\prime}, \phi, x, X^{*}\right)$ is compact (i.e., that the group $\mathbf{G}^{\prime} / Z(\mathbf{G})$ is $k$-anisotropic), so that the character $\Theta_{\pi^{\prime}}$, hence also the function $\Phi_{\pi^{\prime}}$ of Definition 4.1.6, is defined on all of $G^{\prime}$.

Lemma 4.6.1. If $\gamma_{<r} \in G^{\prime}$ and $Y \in \operatorname{Lie}\left(C_{G}^{(r)}(\gamma)\right)$, then we have that

$$
\begin{aligned}
& \underbrace{\varepsilon(\phi, \gamma) \mathfrak{G}(\phi, \gamma)}_{\text {(I) }} \times \\
& {\left[\llbracket \gamma ; x, r \rrbracket: \llbracket \gamma ; x, r \rrbracket_{G^{\prime}} G_{x, r / 2}\right]^{1 / 2}\left[\llbracket \gamma_{<r} ; x, r+\rrbracket: \llbracket \gamma_{<r} ; x, r+\rrbracket_{G^{\prime}} G_{x,(r / 2)+}\right]^{1 / 2} \times} \\
& \underbrace{\Theta_{\pi^{\prime}}\left(\gamma_{<r}\right)}_{\text {(II) }} \underbrace{\hat{\mu}_{X^{*}}^{(r)}(\gamma)}_{\text {(II) }}(Y)
\end{aligned}
$$

(in the notation of [4. Propositions 3.8 and 5.2.13] and [3, Definition 6.6], and with the normalisation of measure of [4, Corollary 6.6]) equals

$$
\begin{aligned}
& \left|D_{G}^{\mathrm{red}}(\gamma)\right|^{-1 / 2}\left|D_{C_{G}^{(r)}(\gamma)}^{\mathrm{red}}(Y)\right|^{-1 / 2} \times \\
& \underbrace{\left[C_{G^{\prime}}\left(\gamma_{<r}\right): C_{G^{\prime}}^{(r)}(\gamma)\right]^{-1}}_{\left(\mathrm{III}_{1}^{\prime}\right)} \underbrace{\varepsilon_{\mathrm{symm}, \mathrm{ram}}\left(\pi^{\prime}, \gamma\right) \varepsilon^{\mathrm{ram}}\left(\pi^{\prime}, \gamma\right)}_{\left(\mathrm{I}_{1}^{\prime}\right)} \underbrace{\Phi_{\pi^{\prime}}(\gamma)}_{\left(\mathrm{II}^{\prime}\right)} \times \\
& \underbrace{\tilde{e}\left(\pi^{\prime}, \gamma\right)}_{\left(\mathrm{I}_{2}^{\prime}\right)} \underbrace{\sum_{h \in C_{G}(\gamma<r) / C_{G}^{(r)}(\gamma)} \hat{O}_{\mathrm{Ad}^{*}(h)^{-1} X^{*}}^{C_{(}^{(r)}(\gamma)}(Y)}_{\left(\mathrm{III}_{2}^{\prime}\right)}
\end{aligned}
$$

(in the notation of Definitions 2.2.8 4.1.6. and 4.3.1).

Proof. As in $\S 4.44 .5$ put $\mathbf{H}=C_{\mathbf{G}}\left(\gamma_{<r}\right)$ and $\mathbf{H}^{\prime}=C_{\mathbf{G}^{\prime}}\left(\gamma_{<r}\right)$, so that $C_{\mathbf{G}}^{(r)}(\gamma)=\mathbf{H}^{\circ}$ and $C_{\mathbf{G}^{\prime}}^{(r)}(\gamma)=\mathbf{H}^{\prime \circ}\left[3\right.$, Corollary 6.14]. (As in 4.1 we have abbreviated $C_{\mathbf{G}^{\prime}}^{(r)}\left(\gamma_{<r}\right)$ to $\left.C_{\mathbf{G}^{\prime}}^{(r)}(\gamma).\right)$ 
By Proposition 4.3.8, $(\mathrm{I})=\left(\mathrm{I}_{1}^{\prime}\right) \cdot\left(\mathrm{I}_{2}^{\prime}\right)$. By Definition 4.1.6 $(\mathrm{II})=\left|D_{G^{\prime}}^{\mathrm{red}}\left(\gamma_{<r}\right)\right|^{-1 / 2}\left(\mathrm{II}^{\prime}\right)$. By Proposition 4.5.1.

$$
(\mathrm{III})=\left|\left(\mathfrak{h}^{\prime}, \mathfrak{h}\right)_{x,(0,0):(0,0+)}\right|^{1 / 2}\left|D_{H}^{\mathrm{red}}\left(X^{*}\right)\right|^{-1 / 2}\left|D_{H}^{\mathrm{red}}(Y)\right|^{-1 / 2}\left(\mathrm{IIII}_{1}^{\prime}\right) \cdot\left(\mathrm{IIII}_{2}^{\prime}\right) .
$$

The result then follows from Corollary 4.2.2.

We are now ready to re-write the character formulæ of [4] in what seems to us to be nearly the optimal form. With the exception of Hypothesis 3.1.2, which has not been needed so far, the hypotheses of Theorem 4.6.2 are the same ones that have been in force throughout the section; we simply re-capitulate them here for convenient reference. Remember that we require $\mathbf{G}$ to satisfy [3, Hypotheses B and C].

Theorem 4.6.2. Suppose that the group $\mathbf{G}$ satisfies Hypothesis 3.1.2] (in addition to [3], Hypotheses B and C]). Let $\left(G^{\prime}, \pi^{\prime}, \phi, x, X^{*}\right)$ be a depth-r, compact, central, expanded cuspidal quintuple in $G$. Suppose that $\gamma=\gamma_{<r} \gamma_{\geq r}$ is regular, semisimple, and $r$-approximable, and put $Y_{\geq r}=\mathrm{e}_{\mathbf{T}, x}^{-1} \gamma_{\geq r}$ for some $k^{\text {tame }}$-split maximal $G$-torus $\mathbf{T}$ such that $\gamma_{<r} \in T$ and $x \in \mathcal{B}(\mathbf{T}, k)$. Then

$$
\begin{gathered}
\Phi_{\mathrm{Y}_{G^{\prime}}^{G} \pi^{\prime}}(\gamma)=\sum_{\substack{(L, \chi) \in C_{G}^{(r)}(\gamma) \backslash G \cdot\left(G^{\prime}, \pi^{\prime}\right) \\
\gamma<r \in L}} \varepsilon_{\mathrm{symm}, \mathrm{ram}}\left(\chi, \gamma_{<r}\right) \cdot \varepsilon^{\mathrm{ram}}\left(\chi, \gamma_{<r}\right) \Phi_{\chi}\left(\gamma_{<r}\right) \times \\
\tilde{e}\left(\chi, \gamma_{<r}\right) \hat{O}_{\chi}^{C_{G}^{(r)}(\gamma)}\left(Y_{\geq r}\right) .
\end{gathered}
$$

Proof. As in the proof of Lemma 4.6.1, put $\mathbf{H}=C_{\mathbf{G}}\left(\gamma_{<r}\right)$. Recall that the connected group $C_{\mathbf{G}}^{(r)}(\gamma)$ is the identity component of $\mathbf{H}$ [3, Corollary 6.14].

For every $g \in G$, we have that $g^{-1} \cdot\left(G^{\prime}, \pi^{\prime}, \phi, x, X^{*}\right)$ is a central, expanded cuspidal $G$ quintuple. Thus, if $g_{1}, g_{2} \in G$ satisfy $g_{1}^{-1} \cdot\left(G^{\prime}, \pi^{\prime}\right)=g_{2}^{-1} \cdot\left(G^{\prime}, \pi^{\prime}\right)$, then, by Remark 4.1.3, we have that $\operatorname{Ad}^{*}\left(g_{1}\right)^{-1} X^{*} \equiv \operatorname{Ad}^{*}\left(g_{2}\right)^{-1} X^{*}\left(\bmod \mathfrak{g}_{x,(-r)+}\right)$. By [29, Lemma 8.3], this implies that $g_{1} \in G^{\prime} g_{2}$. Thus, the map $G^{\prime} \backslash G \rightarrow G \cdot\left(G^{\prime}, \pi^{\prime}\right)$ given by $G^{\prime} g \mapsto g^{-1} \cdot\left(G^{\prime}, \pi^{\prime}\right)$ is a bijection. Since this map is equivariant for the natural (right) $G$-action on both spaces, it induces a bijection $G^{\prime} \backslash G / H^{\circ} \rightarrow H^{\circ} \backslash G \cdot\left(G^{\prime}, \pi^{\prime}\right)$.

By [4, Theorem 6.4 and Proposition 5.3.3] and Corollary 4.4.3, using the notation of Definition 4.4.1, we have that $\Theta_{Y_{G^{\prime}}^{G} \pi^{\prime}}(\gamma)$ is the sum over $g \in G^{\prime} \backslash \mathcal{C}\left(\pi^{\prime}, \gamma_{<r}\right) / H$ of the quantity denoted in [4] by

$$
\begin{aligned}
& \varepsilon\left(\phi, \delta_{<r}\right) \mathfrak{G}\left(\phi, \delta_{<r}\right) \times \\
& {\left[\llbracket \delta_{<r} ; x, r \rrbracket: \llbracket \delta_{<r} ; x, r \rrbracket_{G^{\prime}} G_{x, r / 2}\right]^{1 / 2}\left[\llbracket \delta_{<r} ; x, r+\rrbracket: \llbracket \delta_{<r} ; x, r+\rrbracket_{G^{\prime}} G_{x,(r / 2)+}\right]^{1 / 2} \times} \\
& \Theta_{\pi^{\prime}}\left(\delta_{<r}\right) \hat{\mu}_{X^{*}}^{C_{G}\left(\delta_{<r}\right)}\left(\mathrm{e}_{\mathbf{T}, x}^{-1} \delta_{\geq r}\right),
\end{aligned}
$$

where $\delta=\operatorname{Int}(g) \gamma$ (so that $\delta_{<r}=\operatorname{Int}(g) \gamma_{<r}$ and $\delta_{\geq r}=\operatorname{Int}(g) \gamma_{\geq r}$ ). (Our $\pi^{\prime}$ corresponds to $\pi^{\prime} \otimes \phi$, in the notation of [4].) Note that $\delta_{<r}=\operatorname{Int}(g) \gamma_{<r}$ and $\delta_{\geq r}=\operatorname{Int}(g) \gamma_{\geq r}$. By Lemmata 4.6.1 and 4.1.9, Remark 4.3.5, and Hypothesis 3.1.2, we may re-write the summand as

$$
\begin{aligned}
& \left|D_{G}^{\mathrm{red}}(\gamma)\right|^{-1 / 2} \cdot \varepsilon_{\text {symm, } \operatorname{ram}}\left(\chi, \gamma_{<r}\right) \cdot \varepsilon^{\mathrm{ram}}\left(\chi, \gamma_{<r}\right) \Phi_{\chi}\left(\gamma_{<r}\right) \times \\
& \quad\left[L \cap H:(L \cap H)^{\circ}\right]^{-1} \sum_{h \in H / H^{\circ}} \tilde{e}\left(\chi, \gamma_{<r}\right) \hat{O}_{\mathrm{Ad}^{*}(h)^{-1} X^{\prime *}}^{H^{\circ}}\left(Y_{\geq r}\right),
\end{aligned}
$$

where $\left(L, \chi, X^{\prime *}\right)=g^{-1} \cdot\left(G^{\prime}, \pi^{\prime}, X^{*}\right)$. 
Now write $\widetilde{\mathcal{C}}=\left\{g \in G \mid \operatorname{Int}(g) \gamma_{<r} \in G^{\prime}\right\}$. For any $g \in \widetilde{\mathcal{C}}$, we have that $g^{-1} \cdot\left(G^{\prime}, \pi^{\prime}, \phi, x, X^{*}\right)$ is a central, expanded cuspidal $G$-quintuple, hence, with the notation of Definition 4.1.11, that

$$
\hat{O}_{\mathrm{Ad}^{*}(g)^{-1} X^{*}}^{H^{\circ}}=\hat{O}_{\pi^{\prime} \mathrm{oInt}(g)}^{H^{\circ}} \text {. }
$$

Further, the expression $(\circledast$ is defined, and, by [4, Lemma B.4 and Hypothesis A.7(2)], equals 0 if $g \notin \mathcal{C}$; so $\Theta_{\pi}(\gamma)$ is also the sum of $(\circledast)$ over $\widetilde{\mathcal{C}}$.

Now an elementary counting argument, together with Remark 4.1.10, shows that

$$
\sum_{g \in G^{\prime} \backslash G / H}\left[\operatorname{Int}(g)^{-1} G^{\prime} \cap H:\left(\operatorname{Int}(g)^{-1} G^{\prime} \cap H\right)^{\circ}\right]^{-1} \sum_{h \in H / H^{\circ}} F(g h)=\sum_{g \in G^{\prime} \backslash G / H^{\circ}} F(g)
$$

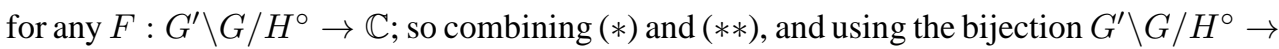
$H^{\circ} \backslash G \cdot\left(G^{\prime}, \pi^{\prime}\right)$, gives the desired result.

\section{STABLE CHARACTER SUMS}

Throughout this section, fix a depth- $r$, toral, cuspidal pair $(T, \phi)$. This pair plays the same rôle as the pair $\left(G^{\prime}, \pi^{\prime}\right)$ did in 44 , but note that the requirements that the pair be compact (Definition 4.1.1) and central (Definition 4.1.4) are now redundant. We will soon require (in Theorems 5.8 and 5.10) that $\mathbf{T}$ is $k^{\mathrm{un}}$-split, but we do not need to do so yet.

Also fix, until Theorem 5.10, a regular, semisimple, $r$-approximable element $\gamma=$ $\gamma_{<r} \gamma_{\geq r}$, and put $\mathbf{H}=C_{\mathbf{G}}^{(r)}(\gamma)$ (i.e., not the possibly disconnected group $C_{\mathbf{G}}\left(\gamma_{<r}\right)$ of 4.4 but its identity component [3, Corollary 6.14], as in 4.1 .

We are almost in a position to show that certain character sums are stable (see Theorem 5.10), but we need two technical preliminaries. First, we show in Lemma5.1 that the definition of $\widehat{S O}_{X^{*}}^{H}$ as a sum (Definition 4.1.6) may be translated into an analogous definition of $\widehat{S O}_{\phi}^{H}$ (Definition 4.1.11). Next, we show in Lemma 5.2 that normal approximations behave well under stable conjugacy.

Lemma 5.1. Write $\mathbf{H} \cdot(T, \phi)$ for the set of stable $\mathbf{H}$-conjugates of $(T, \phi)[10, \S 9.4$, p. 850]. Then

$$
\widehat{S O}_{\phi}^{H}=\sum_{(S, \theta) \in H \backslash \mathbf{H} \cdot(T, \phi)} \hat{O}_{\theta}^{H}
$$

(as functions on $\mathfrak{h}^{\text {rss }} \cap \mathfrak{h}_{r}$ ).

Proof. Recall that $X^{*}$ is an element of $\mathfrak{t}_{-r}^{*}$ that realises $\phi$ on $T_{r / 2}$. By [29, Lemma 8.3] (and the remark on base change at the top of [29, p. 597]), the stabiliser of $X^{*}+$ $\mathfrak{t}\left(k^{\text {un }}\right)_{(-r)+}^{*}$, hence a fortiori of $X^{*}$, in $\mathbf{H}\left(k^{\text {un }}\right)$ is $\mathbf{T}\left(k^{\text {un }}\right)$. In particular, $X^{*}$ is strongly regular semisimple. Given this, and the fact that $C_{\mathbf{H}}(\mathbf{T})=\mathbf{T}$ [6, Corollary 13.17(2)], it is a straightforward check that, for $g \in \mathbf{H}\left(k^{\text {un }}\right)$, we have that $\operatorname{Ad}^{*}(g) X^{*}$ is defined over $k$ if and only if both the group $\operatorname{Int}(g) \mathbf{T}$ and the map $\operatorname{Int}(g): \mathbf{T} \rightarrow \operatorname{Int}(g) \mathbf{T}$ are defined over $k$. Fix such an element $g$, and put $\mathbf{T}^{\prime}=\operatorname{Int}(g) \mathbf{T}$.

The Moy-Prasad map for $\mathbf{T}$ (at depth $(r / 2)+$ ) is defined to be the unique isomorphism $\mathrm{e}_{(r / 2)+: r+}: \mathfrak{t}_{(r / 2)+: r+} \rightarrow T_{(r / 2)+: r+}$ such that, if $Y \in \mathfrak{t}_{(r / 2)+}$ and $\gamma \in \mathrm{e}_{(r / 2)+: r+}(Y)$, then

$$
\operatorname{ord}((\chi(\gamma)-1)-\mathrm{d} \chi(Y))>r
$$

for all characters $\chi$ of $\mathbf{T}_{k^{\text {sp }}}[1, \S 1.5$, p. 11]. Then

$$
\mathrm{e}_{(r / 2)+: r+}^{\prime}:=\operatorname{Int}(g) \circ \mathrm{e}_{(r / 2)+: r+} \circ \operatorname{Ad}(g)^{-1}
$$


satisfies the analogous property for $\mathbf{T}^{\prime}$. By the definition of 'realise', if $Y \in \mathfrak{t}_{(r / 2)+}$ and $\gamma \in \mathrm{e}_{(r / 2)+: r+}(Y)$, then

$(* *) \quad\left(\phi \circ \operatorname{Int}(g)^{-1}\right)(\operatorname{Int}(g) \gamma)=\phi(\gamma)=\Lambda\left(\left\langle X^{*}, Y\right\rangle\right)=\Lambda\left(\left\langle\operatorname{Ad}^{*}(g) X^{*}, \operatorname{Ad}(g) Y\right\rangle\right)$.

Since $\operatorname{Ad}(g)$ carries $\mathfrak{t}_{(r / 2)+}$ and $\mathfrak{t}_{r+}$ onto $\mathfrak{t}_{(r / 2)+}^{\prime}$ and $\mathfrak{t}_{r+}^{\prime}$, respectively, it follows from (ख) and (**), and the definition of 'realise' again, that $\operatorname{Ad}^{*}(g) X^{*}$ realises $\phi \circ \operatorname{Int}(g)^{-1}$ on $\mathfrak{t}_{(r / 2)+}^{\prime}$. In particular, since we have already observed that the stabiliser of $X^{*}+\mathfrak{t}\left(k^{\mathrm{un}}\right)_{(-r)+}$ in $\mathbf{H}\left(k^{\text {un }}\right)$ is $\mathbf{T}\left(k^{\text {un }}\right)$, it follows that $\phi \circ \operatorname{Int}(g)^{-1}=\phi$ (if and) only if $g \in \mathbf{T}\left(k^{\text {un }}\right)$.

In all, we have shown that $\left(\operatorname{Int}(g) T, \phi \circ \operatorname{Int}(g)^{-1}\right) \mapsto \operatorname{Ad}^{*}(g) X^{*}$ is a bijection from the $\mathbf{H}$-stable conjugacy class of $(T, \phi)$ to $\operatorname{Ad}^{*}\left(\mathbf{H}\left(k^{\text {un }}\right)\right) X^{*} \cap \mathfrak{g}^{*}$ such that, if $\left(T^{\prime}, \phi^{\prime}\right)$ has image $X^{\prime *}$, then $\phi^{\prime}$ is represented on $\mathfrak{t}_{(r / 2)+}^{\prime}$ by $X^{\prime *}$; in which case, in the notation of Definition 4.1.11, we have that $\hat{O}_{\phi^{\prime}}^{H}=\hat{O}_{X^{\prime *}}^{H}$. Since the bijection is $H$-equivariant, the desired equality follows.

\section{Lemma 5.2. If}

- $\mathbf{S}$ is a $k^{\text {tame }}$-split maximal $G$-torus,

- $\gamma \in S$,

- $\left(\gamma_{i}\right)_{0 \leq i<r}$ is a normal r-approximation to $\gamma$ in $G$ [3, Definition 6.8], and

- $g \in \mathbf{G}\left(k^{\text {sep }}\right)$ is such that $g^{-1} \sigma g \in \mathbf{S}\left(k^{\text {sep }}\right)$ for all $\sigma \in \Gamma$,

then $\left(\operatorname{Int}(g) \gamma_{i}\right)_{0 \leq i<r}$ is a normal r-approximation to $\operatorname{Int}(g) \gamma$ in $G$.

Remark 5.3. Under the hypotheses of Lemma 5.2 $\delta:=\operatorname{Int}(g) \gamma$ is $k$-rational. If $\gamma$ is a strongly regular semisimple element of $G$, then the $k$-rationality of $\delta$ is equivalent to the hypothesis on $g$.

Proof. Recall that $\gamma_{<r}=\prod_{0 \leq i<r} \gamma_{i}$ and $\gamma_{\geq r}=\prod_{i \geq r} \gamma_{i}$ [3, §6, p. 52].

The group $\mathbf{S}^{\prime}:=\operatorname{Int}(g) \mathbf{S}$ and the map $\operatorname{Int}(g): \mathbf{S} \rightarrow \mathbf{S}^{\prime}$ are defined over $k$. In particular, $\mathbf{S}^{\prime}$ is a $k^{\text {tame }}$-split, maximal $G$-torus, and the set $S^{\prime}$ of $k$-rational elements of $\mathbf{S}^{\prime}$ is precisely $\operatorname{Int}(g) S$.

Fix an index $i$ with $0 \leq i<r$. Since $\gamma_{i} \in Z(H) \subseteq S$ [3, Remark 6.10(1)], it follows that $\delta_{i}:=\operatorname{Int}(g) \gamma_{i}$ belongs to $S^{\prime}$. If $\delta_{i} \neq 1$, then, because the root values [3, Definition A.4] of $\gamma_{i}$ and $\delta_{i}=\operatorname{Int}(g) \gamma_{i}$ are the same, we have that $\delta_{i}$ is good of depth $i$ [3, Definition 6.1].

That is, $\underline{\delta}:=\left(\delta_{i}\right)_{0 \leq i<r}$ is a good sequence [3, Definition 6.4]. Since $\left(\gamma_{i}\right)_{0 \leq i<r}$ is a normal $r$-approximation to $\gamma$ [3, Definition 6.8], we have that $\gamma_{\geq r}=\prod_{i>r} \gamma_{i} \in G_{y, r} \cap S=$ $S_{r}$ for some $y \in \mathcal{B}(\mathbf{S}, k)$ (see [3, Definition 4.3 and Proposition 4.6]). Thus $\delta_{\geq r}:=$ $\prod_{i \geq r} \delta_{i}=\operatorname{Int}(g) \gamma_{\geq r} \in \operatorname{Int}(g) S_{r}=S_{r}^{\prime} \subseteq C_{G}^{(r)}(\underline{\delta})_{y^{\prime}, r}$, where $y^{\prime}$ is any point in $\mathcal{B}\left(\mathbf{S}^{\prime}, k\right) \subseteq$ $\mathcal{B}\left(C_{\mathbf{G}}^{(r)}(\underline{\delta}), k\right)$; so the result follows from [3, Definition 6.8] again.

It turns out that the original induction map $\mathrm{Y}^{G}$ is not quite suited to Reeder's conjectural construction of L-packets [21, §6.6, p. 18], precisely because of the appearance in Theorem 4.6.2 of the character $\varepsilon^{\mathrm{ram}}(\phi)$. In order to obtain stable character sums from Reeder's construction, we must twist away this (quadratic) character.

Definition 5.4. Put $\tilde{Y}_{T}^{G} \phi=\mathrm{Y}_{T}^{G}\left(\phi \cdot \varepsilon^{\mathrm{ram}}(\phi)^{-1}\right)$, where $\mathrm{Y}_{T}^{G}$ is as in Definition 4.1.2 and $\varepsilon^{\mathrm{ram}}(\phi)$ is the character $\gamma \mapsto \varepsilon^{\mathrm{ram}}(\phi, \gamma)$ of Definition 4.3.1.

Example 5.5. There are cases in which the collection of representations

$$
\left\{\widetilde{Y}_{S}^{G} \theta \mid(S, \theta) \text { is a stable conjugate of }(T, \phi)\right\}
$$


formed by our modified Yu-type construction is different from the collection

$$
\left\{\mathrm{Y}_{S}^{G} \theta \mid(S, \theta) \text { is a stable conjugate of }(T, \phi)\right\}
$$

considered by Reeder.

For $\mathbf{G}=\mathrm{PGSp}_{4}$, there exist stably $\mathbf{G}$-conjugate, $k^{\mathrm{un}}$-split, $k$-anisotropic, maximal $G$-tori $\mathbf{S}$ and $\mathbf{T}$ such that

- Fr acts by inversion on both,

- $\mathcal{B}(\mathbf{T}, k)$ contains a hyperspecial vertex $x$ of $\mathcal{B}(\mathbf{G}, k)$, and

- $\mathcal{B}(\mathbf{S}, k)$ contains a non-hyperspecial vertex $y$ of $\mathcal{B}(\mathbf{G}, k)$,

Let $\mathfrak{f}_{2} / \mathfrak{f}$ be the quadratic extension of $\mathfrak{f}$, so that $\mathfrak{f}_{\alpha}=\mathfrak{f}_{\alpha^{\prime}}=\mathfrak{f}_{2}$ for all roots $\alpha$ of $\mathbf{T}$ in $\mathbf{G}$ and $\alpha^{\prime}$ of $\mathbf{T}^{\prime}$ in $\mathbf{G}$, and $\mathfrak{f}_{2}^{1}$ the kernel of the norm map from $\mathfrak{f}_{2}$ to $\mathfrak{f}$. Let $\{\alpha, \beta\}$ be a system of simple roots for $\mathbf{T}$ in $\mathbf{G}$, with $\alpha$ short; and $\left\{\alpha^{\prime}, \beta^{\prime}\right\}$ the corresponding system of simple roots for $\mathbf{S}$ in $\mathbf{G}$. Since all roots of $\mathbf{S}$ and $\mathbf{T}$ in $\mathbf{G}$ are symmetric, we have that $\varepsilon_{x, r / 2}^{\text {symm }}(G, T, \gamma)=1$ and $\varepsilon_{y, r / 2}^{\text {symm }}\left(G, S, \gamma^{\prime}\right)$ are trivial for all $r, \gamma \in T$, and $\gamma^{\prime} \in S$. Let $\phi$ be any $G$-generic character of $T$ [29, $\S 9$, p. 599], and $\theta$ the corresponding character of $S$. The common depth of $\phi$ and $\theta$ is a positive integer $r$.

If $r$ is even, then $R(\mathbf{G}, \mathbf{T})_{x, r / 2, \text { symm, unram }}=R(\mathbf{G}, \mathbf{T})$ and $R(\mathbf{G}, \mathbf{S})_{y, r / 2 \text {, symm, unram }}=$ $\left\{ \pm \beta^{\prime}, \pm\left(2 \alpha^{\prime}+\beta^{\prime}\right)\right\}$, so that $\widetilde{Y}_{T}^{G} \phi=\mathrm{Y}_{S}^{G}\left(\phi\left(\operatorname{sgn}_{\mathfrak{f}_{2}^{1}} \circ \beta\right)^{-1}\right)$ and $\widetilde{Y}_{S}^{G} \theta=\mathrm{Y}_{T}^{G} \theta$. If $\gamma=$ $\rho^{\vee}(t) \in T$, where $\rho^{\vee}=\frac{3}{2} \alpha^{\vee}+2 \beta^{\vee}$ is the half-sum of the positive co-roots of $\mathbf{T}$ in $\mathbf{G}$ (with respect to the chosen system of simple roots) and $t$ is a generator of $\mathfrak{f}_{2}^{1}$, then $\operatorname{sgn}_{\mathfrak{f}_{2}^{1}}(\beta(\gamma))=\operatorname{sgn}_{\mathfrak{f}_{2}^{1}}(t)=-1$.

If $r$ is odd, then $R(\mathbf{G}, \mathbf{T})_{x, r / 2 \text {, symm, unram }}=\emptyset$ and $R(\mathbf{G}, \mathbf{S})_{y, r / 2 \text {, symm, unram }}=\left\{ \pm \alpha^{\prime}, \pm\left(\alpha^{\prime}+\beta^{\prime}\right)\right\}$, so that $\tilde{Y}_{T}^{G} \phi=\mathrm{Y}_{T}^{G} \phi$ and $\widetilde{Y}_{S}^{G} \theta=\mathrm{Y}_{S}^{G}\left(\theta\left(\operatorname{sgn}_{\mathfrak{f}_{2}^{1}} \circ \beta^{\prime}\right)^{-1}\right)$; and, with the obvious notation, $\operatorname{sgn}_{\mathfrak{f}_{2}^{1}}\left(\beta^{\prime}\left(\rho^{\prime \vee}(t)\right)\right)=-1$.

Since the characters $\operatorname{sgn}_{\mathfrak{f}_{2}^{1}} \circ \beta$ and $\operatorname{sgn}_{\mathfrak{f}_{2}^{1}} \circ \beta^{\prime}$ are trivial on the subgroups of $T$ and $S$, respectively, consisting of elements that admit a lift in $\operatorname{Sp}_{4}(k)$, we really must work on $\mathrm{PGSp}_{4}(k)$ to see this phenomenon.

Corollary 5.6 (to Theorem 4.6.2). With the notation of Theorem 4.6.2

$$
\Phi_{\widetilde{Y}_{T}^{G} \phi}(\gamma)=\sum_{(S, \theta) \in C_{G}^{(r)}(\gamma) \backslash G \cdot(T, \phi)} \varepsilon_{\substack{\gamma_{<r} \in S \\ \text { symm, ram }}}\left(\theta, \gamma_{<r}\right) \cdot \theta\left(\gamma_{<r}\right) \cdot \tilde{e}\left(\theta, \gamma_{<r}\right) \hat{O}_{\theta}^{H}\left(Y_{\geq r}\right) .
$$

Remark 5.7. With the notation of Corollary 5.6 the formula there shows that $\Phi_{\widetilde{Y}_{T}^{G} \phi}(\gamma)$ vanishes unless the $G$-orbit of $\gamma_{<r}$ intersects $T$; so, for definiteness, we could require that actually $\gamma_{<r} \in T$, and then take $\mathbf{T}$ as the torus used to define of $Y_{\geq r}$ in the statement of Theorem 4.6.2

As we did for Theorem 4.6.2 we re-capitulate in Theorems 5.8 and 5.10 below the standing hypotheses of this section. Remember that we require $\mathbf{G}$ to satisfy [3, Hypotheses $\mathrm{B}$ and $\mathrm{C}]$.

Theorem 5.8. Suppose that the group $\mathbf{G}$ satisfies Hypothesis 3.1 .2 (in addition to [3, Hypotheses B and C]). Let $(T, \phi)$ be a depth-r, toral, cuspidal pair, and suppose that $\mathbf{T}$ is $k^{\mathrm{un}}$-split. Suppose that $\gamma=\gamma_{<r} \gamma_{\geq r}$ is regular, semisimple, and $r$-approximable, and that $\gamma_{<r} \in T$, and put $Y_{\geq r}=\mathrm{e}_{\mathbf{T}, x}^{-1} \gamma_{\geq r}$. As in Lemma 5.1] write $\mathbf{G} \cdot(T, \phi)$ for the set of stable conjugates of $(T, \phi)$. Then

$$
(-1)^{\mathrm{rank}_{k} \mathbf{G}} \sum_{(S, \theta) \in G \backslash \mathbf{G} \cdot(T, \phi)} \Phi_{\widetilde{Y}_{S}^{G} \theta}(\gamma)
$$


equals

$$
\begin{array}{r}
\sum_{\substack{(S, \theta) \in C_{\mathbf{G}}^{(r)}(\gamma) \backslash \mathbf{G} \cdot(T, \phi) \\
\gamma<r \in S}} \varepsilon_{\text {symm, ram }}\left(\theta, \gamma_{<r}\right) \cdot \theta\left(\gamma_{<r}\right) \cdot e\left(\theta, \gamma_{<r}\right) \times \\
(-1)^{\text {rank }_{k} C_{\mathbf{G}}^{(r)}(\gamma) \cdot \widehat{S O}_{\theta}^{C(r)}(\gamma)}\left(Y_{\geq r}\right),
\end{array}
$$

where $C_{\mathbf{G}}^{(r)}(\gamma) \backslash \mathbf{G} \cdot(T, \phi)$ is the set of $C_{\mathbf{G}}^{(r)}(\gamma)$-stable conjugacy classes in $\mathbf{G} \cdot(T, \phi)$.

Note that the indexing sets for the two sums are different.

Remark 5.9. As in Remark 5.7 the assumption that $\gamma_{<r} \in T$ is just for convenience. If the $\mathbf{G}\left(k^{\text {un }}\right)$-conjugacy class of $\gamma_{<r}$ does not intersect $T$, then the set of stable Gconjugates $(S, \theta)$ of $(T, \phi)$ with $\gamma_{<r} \in S$ is empty, and Corollary 5.6 shows that the function $(-1)^{\mathrm{rank}_{k} \mathrm{G}} \sum \Phi_{\widetilde{Y}_{S}^{G} \theta}$ of Theorem 5.8 vanishes at $\gamma$.

Proof. Put $\mathbf{H}=C_{\mathbf{G}}^{(r)}(\gamma)$. By Corollary 5.6 and Proposition 4.3.7.

$$
(-1)^{\mathrm{rank}_{k} \mathbf{G}} \sum_{(S, \theta) \in G \backslash \mathbf{G} \cdot(T, \phi)} \Phi_{\widetilde{Y}_{S}^{G} \theta}(\gamma)
$$

is equal to the summation

$$
\begin{aligned}
& \sum_{(S, \theta) \in G \backslash \mathbf{G} \cdot(T, \phi)} \sum_{\substack{\left(S^{\prime}, \theta^{\prime}\right) \in H \backslash G \cdot(S, \theta) \\
\gamma<r \in S^{\prime}}} F\left(\theta^{\prime}\right) \\
& \begin{array}{ll}
= & \sum_{\substack{\left(S^{\prime}, \theta^{\prime}\right) \in H \backslash \mathbf{G} \cdot(T, \phi) \\
\gamma<r \in S^{\prime}}} \sum_{\substack{(S, \theta) \in \mathbf{H} \backslash \mathbf{G} \cdot(T, \phi)}} F\left(\theta^{\prime}\right) \\
\substack{\left(S^{\prime}, \theta^{\prime}\right) \in H \backslash \mathbf{H} \cdot(S, \theta) \\
\gamma_{<r} \in S^{\prime}} & F\left(\theta^{\prime}\right),
\end{array}
\end{aligned}
$$

where $\mathbf{H} \backslash \mathbf{G} \cdot(T, \phi)$ is the set of stable G-conjugacy classes in $\mathbf{G} \cdot(T, \phi), \mathbf{H} \cdot(S, \theta)$ is the set of stable $\mathbf{H}$-conjugates of $(S, \theta)$, and

$$
F\left(\theta^{\prime}\right):=\varepsilon_{\text {symm, } \operatorname{ram}}\left(\theta^{\prime}, \gamma_{<r}\right) \cdot \Phi_{\theta^{\prime}}\left(\gamma_{<r}\right) \cdot e\left(\theta^{\prime}, \gamma_{<r}\right) \cdot(-1)^{\mathrm{rank}_{k} \mathbf{H}} \hat{O}_{\theta^{\prime}}^{H}\left(Y_{\geq r}\right)
$$

for $\left(S^{\prime}, \theta^{\prime}\right) \in \mathbf{H} \cdot(S, \theta)$. Note that, in the above notation, the condition $\gamma_{<r} \in S^{\prime}$, the number $\Phi_{\theta^{\prime}}\left(\gamma_{<r}\right)$, and (by Lemma 4.3.6) the numbers $\varepsilon_{\text {symm, ram }}\left(\theta^{\prime}, \gamma_{<r}\right)$ and $e\left(\theta^{\prime}, \gamma_{<r}\right)$ depend only on $(S, \theta)$, not $\left(S^{\prime}, \theta^{\prime}\right)$; and that, by Definitions 2.2.8 and 4.1.6 the function $D_{S}^{\text {red }}$ is identically 1 , so that $\Phi_{\theta}=\theta$. The result now follows from Lemma 5.1

Theorem 5.10. Suppose that $\mathbf{G}$ satisfies [10, Restriction 12.4.1(2)] (in addition to [3, Hypotheses B and C]). Let $(T, \phi)$ be a positive-depth, toral, cuspidal pair, and suppose that $\mathbf{T}$ is $k^{\mathrm{un}}$-split. Then

$$
\Theta_{\widetilde{Y}_{T}^{G} \phi}^{\mathrm{st}}:=\sum_{(S, \theta) \in G \backslash \mathbf{G} \cdot(T, \phi)} \Theta_{\widetilde{Y}_{S}^{G} \theta}
$$

is stable on the set of $r$-approximable and strongly regular semisimple elements of $G$. That is, it is constant on each stable conjugacy class (see [17, §3, p. 788] and [10, §2.9, p. 817]) in that set. 
Remark 5.11. By [3, Lemma 8.1], under mild conditions, every element of a tame torus is $r$-approximable. Of course, if $p$ is sufficiently large, then every semisimple element of $G$ lies in a tame torus.

Proof. By [10, Lemma B.0.3], the exponential map e converges on $\mathfrak{g}\left(k^{\mathrm{un}}\right)_{0+}$; so we may, and do, take the maps $\mathrm{e}_{\mathbf{T}, \bar{x}}$ of Hypothesis 3.1.2 to be restrictions of $\mathrm{e}$.

Let $\gamma=\gamma_{<r} \gamma_{\geq r}$ be an $r$-approximable and strongly regular semisimple element of $G$. By Theorem 5.8, $\Theta_{\widetilde{Y}_{S}^{G} \theta}^{\mathrm{st}}(\gamma)=0$ unless the $\mathbf{G}\left(k^{\text {un }}\right)$-orbit of $\gamma_{<r}$ intersects $T$.

Thus, we may, and do, suppose that $\gamma_{<r} \in T$, and then show that

$$
\Theta_{\widetilde{Y}_{S}^{G} \theta}^{\mathrm{st}}(\gamma)=\Theta_{\widetilde{Y}_{S}^{G} \theta}^{\mathrm{st}}(\operatorname{Int}(g) \gamma)
$$

for any element $g \in \mathbf{G}\left(k^{\mathrm{un}}\right)$ such that $\operatorname{Int}(g) \gamma$ is defined over $k$, i.e., such that $g^{-1} \operatorname{Fr} g \in$ $C_{\mathbf{G}}(\gamma)\left(k^{\text {un }}\right)$.

Given such an element $g$, put $\gamma^{\prime}=\operatorname{Int}(g) \gamma$ and $\mathbf{T}^{\prime}=\operatorname{Int}(g) \mathbf{T}$. As usual, note that $\mathbf{T}^{\prime}$ is defined over $k$, and $\gamma^{\prime} \in T^{\prime}$. By Lemma 5.2, the element $\gamma^{\prime}$ is also $r$-approximable, with $\gamma_{<r}^{\prime}=\operatorname{Int}(g) \gamma_{<r}$ and $\gamma_{\geq r}^{\prime}=\operatorname{Int}(g) \gamma_{\geq r}$. Put $Y_{\geq r}^{\prime}=\mathrm{e}^{-1} \gamma_{\geq r}^{\prime}$. Put $\mathbf{J}=C_{\mathbf{G}}^{(r)}\left(\gamma^{\prime}\right)=$ $\operatorname{Int}(g) \mathbf{H}$ [3, Corollary 6.14]. Exactly as in [10, $\$ 11.1$, p. 861], there is a bijection

$$
\iota_{g}: \mathbf{H} \backslash\left\{(S, \theta) \in \mathbf{G} \cdot(T, \phi) \mid \gamma_{<r} \in S\right\} \rightarrow \mathbf{J} \backslash\left\{\left(S^{\prime}, \theta^{\prime}\right) \in \mathbf{G} \cdot(T, \phi) \mid \gamma_{<r}^{\prime} \in S^{\prime}\right\},
$$

where, as usual, $\mathbf{G} \cdot(T, \phi)$ is the set of stable $\mathbf{G}$-conjugates of $(T, \phi)$, and the notations $\mathbf{H} \backslash \cdots$ and $\mathbf{J} \backslash \cdots$ stand for the collections of stable $\mathbf{H}$ - and $\mathbf{J}$-conjugacy classes, respectively, in the appropriate sets. If $\iota_{g}(S, \theta)=\left(S^{\prime}, \theta^{\prime}\right)$, then

- by construction, we have that $\theta\left(\gamma_{<r}\right)=\theta^{\prime}\left(\gamma_{<r}^{\prime}\right)$;

- by Lemma 4.3.6, we have that $\varepsilon_{\mathrm{symm}, \mathrm{ram}}\left(\theta, \gamma_{<r}\right)=\varepsilon_{\mathrm{symm}, \mathrm{ram}}\left(\theta^{\prime}, \gamma_{<r}^{\prime}\right)$ and $e\left(\theta, \gamma_{<r}\right)=$ $e\left(\theta^{\prime}, \gamma_{<r}^{\prime}\right)$; and

- by [10, Lemma 12.2.3], we have that

$$
(-1)^{\mathrm{rank}_{k} \mathbf{J}} \cdot \widehat{S O}_{\theta^{\prime}}^{J}\left(Y_{\geq r}^{\prime}\right)=(-1)^{\mathrm{rank}_{k} \mathbf{H}} \cdot \widehat{S O}_{\theta}^{H}\left(Y_{\geq r}\right) .
$$

(See [10, Example 12.2.1].)

The equality ( $\circledast$ ) now follows from Theorem 5.8

\section{REFERENCES}

[1] Jeffrey D. Adler, Refined anisotropic K-types and supercuspidal representations, Pacific J. Math. 185 (1998), no. 1, 1-32. MR1653184 (2000f:22019)

[2] Jeffrey D. Adler and Stephen DeBacker, Murnaghan-Kirillov theory for supercuspidal representations of tame general linear groups, J. Reine Angew. Math. 575 (2004), 1-35. MR2097545 (2005j:22008)

[3] Jeffrey D. Adler and Loren Spice, Good product expansions for tame elements of p-adic groups, Int. Math. Res. Pap. 2008, no. 1, DOI 10.1093/imrp/rpn003, available at arXiv:math.RT/0611554 MR2431235

[4] __ Supercuspidal characters of reductive p-adic groups, Amer. J. Math. 131 (2009), no. 4, 1136-1210, available at arXiv:0707.3313

[5] Jeffrey D. Adler, Stephen DeBacker, Paul J. Sally Jr., and Loren Spice, Supercuspidal characters of $\mathrm{SL}_{2}$ over a $p$-adic field, Harmonic analysis on reductive, $p$-adic groups, Contemporary Mathematics, vol. 543, American Mathematical Society, Providence, RI, 2011. Edited by Robert S. Doran, Paul J. Sally, Jr. and Loren Spice, pp. 19-70.

[6] Armand Borel, Linear algebraic groups, Graduate Texts in Mathematics, vol. 126, Springer-Verlag, New York, 1991. MR1102012 (92d:20001)

[7] François Bruhat and Jacques Tits, Groupes réductifs sur un corps local, Publ. Math. Inst. Hautes Études Sci. 41 (1972), 5-251 (French). MR0327923 (48 \#6265)

[8] _ Groupes réductifs sur un corps local. II. Schémas en groupes. Existence d'une donnée radicielle valuée, Publ. Math. Inst. Hautes Études Sci. 60 (1984), 197-376 (French). MR756316 (86c:20042) 
[9] Colin J. Bushnell and Guy Henniart, The essentially tame local Langlands correspondence, III: the general case, Proc. Lond. Math. Soc. (3) 101 (2010), no. 2, 497-553, DOI 10.1112/plms/pdp053. MR2679700 (2011j:22023)

[10] Stephen DeBacker and Mark Reeder, Depth-zero supercuspidal L-packets and their stability, Ann. Math. 169 (2009), no. 3, 795-901.

[11] Paul Gérardin, Weil representations associated to finite fields, J. Algebra 46 (1977), no. 1, 54-101. MR0460477 (57 \#470)

[12] Jeffrey Hakim and Fiona Murnaghan, Distinguished tame supercuspidal representations, Int. Math. Res. Pap. IMRP 2 (2008), Art. ID rpn005, 166. MR2431732 (2010a:22022)

[13] Harish-Chandra, Harmonic analysis on reductive p-adic groups, notes by G. van Dijk, Lecture Notes in Mathematics, vol. 162, Springer-Verlag, Berlin, 1970. MR0414797 (54 \#2889)

[14] _ A submersion principle and its applications, Geometry and analysis: Papers dedicated to the memory of V. K. Patodi, Indian Academy of Sciences, Bangalore, 1980, pp. 95-102. MR592255 (82e:22032)

[15] Ju-Lee Kim and Fiona Murnaghan, Character expansions and unrefined minimal K-types, Amer. J. Math. 125 (2003), no. 6, 1199-1234. MR2018660 (2004k:22024)

[16] Ju-Lee Kim, Supercuspidal representations: an exhaustion theorem, J. Amer. Math. Soc. 20 (2007), no. 2, 273-320 (electronic). MR2276772

[17] Robert E. Kottwitz, Rational conjugacy classes in reductive groups, Duke Math. J. 49 (1982), no. 4, 785806. MR683003 (84k:20020)

[18] - Sign changes in harmonic analysis on reductive groups, Trans. Amer. Math. Soc. 278 (1983), no. 1, 289-297, DOI 10.2307/1999316. MR697075 (84i:22012)

[19] Allen Moy and Gopal Prasad, Unrefined minimal K-types for p-adic groups, Invent. Math. 116 (1994), no. 1-3, 393-408. MR1253198 (95f:22023)

[20] __ Jacquet functors and unrefined minimal K-types, Comment. Math. Helv. 71 (1996), no. 1, 98-121. MR1371680 (97c:22021)

[21] Mark Reeder, Supercuspidal L-packets of positive depth and twisted Coxeter elements, J. Reine Angew. Math. 620 (2008), 1-33. MR2427973

[22] Jean-Pierre Serre, Galois cohomology, Springer Monographs in Mathematics, Springer-Verlag, Berlin, 2002 (English). MR1867431 (2002i:12004)

[23] Loren Spice, Sign changes in harmonic analysis on reductive p-adic groups, in preparation.

[24] _ On counting orbits in root systems, Submitted to Representation Theory, available at arXiv:math.RT/1308.4056

[25] Tonny A. Springer, Linear algebraic groups, Progress in Mathematics, vol. 9, Birkhäuser Boston Inc., Boston, MA, 1998. MR1642713 (99h:20075)

[26] Jacques Tits, Reductive groups over local fields, Automorphic forms, representations and $L$-functions. Part 1 (A. Borel and W. Casselman, eds.), Proceedings of Symposia in Pure Mathematics, vol. XXXIII, American Mathematical Society, Providence, R.I., 1979, pp. 29-69. MR546588 (80h:20064)

[27] J.-L. Waldspurger, Le lemme fondamental implique le transfert, Compositio Math. 105 (1997), no. 2, 153236, DOI 10.1023/A:1000103112268 (French). MR1440722 (98h:22023)

[28] Jean-Loup Waldspurger, Intégrales orbitales nilpotentes et endoscopie pour les groupes classiques non ramifiés, Astérisque 269 (2001), vi+449 (French). MR1817880 (2002h:22014)

[29] Jiu-Kang Yu, Construction of tame supercuspidal representations, J. Amer. Math. Soc. 14 (2001), no. 3, 579-622 (electronic). MR1824988 (2002f:22033)

[30] _ The construction of supercuspidals/types revisited. Talk at conference "Characters, liftings, and types", American University, June 2012.

University of Michigan, ANn ARbor, MI 48109

E-mail address: smdbackr@umich.edu

TEXAs Christian UnIVERSITY, Fort WORTH, TX 76129

E-mail address: 1 .spice@tcu.edu 